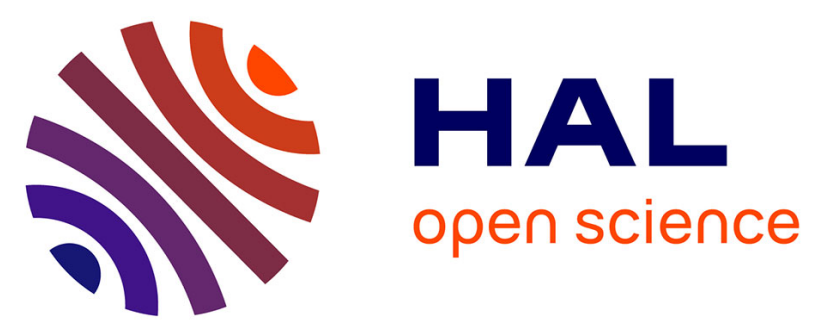

\title{
Pharmacological rescue of nociceptive hypersensitivity and oxytocin analgesia impairment in a rat model of neonatal maternal separation
}

Meggane Melchior, Pierre-Eric Juif, Géraldine Gazzo, Nathalie Petit-Demoulière, Virginie Chavant, Adrien Lacaud, Yannick Goumon, Alexandre Charlet, Vincent Lelièvre, Pierrick Poisbeau

\section{To cite this version:}

Meggane Melchior, Pierre-Eric Juif, Géraldine Gazzo, Nathalie Petit-Demoulière, Virginie Chavant, et al.. Pharmacological rescue of nociceptive hypersensitivity and oxytocin analgesia impairment in a rat model of neonatal maternal separation. Pain, 2018, 159 (12), pp.2630-2640. 10.1097/j.pain.0000000000001375 . hal-02166011

\section{HAL Id: hal-02166011 \\ https://hal.science/hal-02166011}

Submitted on 4 Nov 2020

HAL is a multi-disciplinary open access archive for the deposit and dissemination of scientific research documents, whether they are published or not. The documents may come from teaching and research institutions in France or abroad, or from public or private research centers.
L'archive ouverte pluridisciplinaire HAL, est destinée au dépôt et à la diffusion de documents scientifiques de niveau recherche, publiés ou non, émanant des établissements d'enseignement et de recherche français ou étrangers, des laboratoires publics ou privés. 
PAIN

\section{Pharmacological rescue of nociceptive hypersensitivity and oxytocin analgesia impairment in a rat model of neonatal maternal separation

\author{
--Manuscript Draft--
}

\begin{tabular}{|c|c|}
\hline Manuscript Number: & PAIN-D-17-01200R1 \\
\hline Full Title: & $\begin{array}{l}\text { Pharmacological rescue of nociceptive hypersensitivity and oxytocin analgesia } \\
\text { impairment in a rat model of neonatal maternal separation }\end{array}$ \\
\hline Article Type: & Research Paper \\
\hline Keywords: & $\begin{array}{l}\text { early life stress, spinal nociceptive porcessing, hyperalgesia, oxytocin, } \\
\text { allopregnanolone, HDAC inhibitor, epigenetic }\end{array}$ \\
\hline Corresponding Author: & $\begin{array}{l}\text { Pierrick Poisbeau, PhD } \\
\text { CNRS and University of Strasbourg } \\
\text { Strasbourg, FRANCE }\end{array}$ \\
\hline \multicolumn{2}{|l|}{$\begin{array}{l}\text { Corresponding Author Secondary } \\
\text { Information: }\end{array}$} \\
\hline Corresponding Author's Institution: & CNRS and University of Strasbourg \\
\hline \multicolumn{2}{|l|}{$\begin{array}{l}\text { Corresponding Author's Secondary } \\
\text { Institution: }\end{array}$} \\
\hline First Author: & Meggane Melchior, MSc \\
\hline \multicolumn{2}{|l|}{ First Author Secondary Information: } \\
\hline \multirow[t]{10}{*}{ Order of Authors: } & Meggane Melchior, MSc \\
\hline & Pierre-Eric Juif, PhD \\
\hline & Géraldine Gazzo, MSc \\
\hline & Nathalie Petit-Demouliere, MSc \\
\hline & Virgine Chavant, MSc \\
\hline & Adrien Lacaud, Tech \\
\hline & Yannick Goumon, PhD \\
\hline & Alexandre Charlet, PhD \\
\hline & Vincent Lelièvre, PhD \\
\hline & Pierrick Poisbeau, PhD \\
\hline \multicolumn{2}{|l|}{ Additional Information: } \\
\hline Question & Response \\
\hline $\begin{array}{l}\text { Have you posted this manuscript on a } \\
\text { preprint server (e.g., arXiv.org, BioXriv, } \\
\text { PeerJ Preprints)? }\end{array}$ & No \\
\hline
\end{tabular}




\begin{abstract}
Oxytocin (OT), known for its neurohormonal effects around birth, has recently been suggested for being a critical determinant in neurodevelopmental disorders. This hypothalamic neuropeptide exerts a potent analgesic effect through an action on the nociceptive system. This endogenous control of pain has an important adaptive value but might be altered by early life stress (ELS), possibly contributing to its long-term consequences on pain responses and associated comorbidities. We tested this hypothesis using a rat model of neonatal maternal separation (MS) known to induce long term consequences on several brain functions including chronic stress, anxiety, altered social behavior and visceral hypersensitivity. We found that adult rats with a history of MS were hypersensitive to noxious mechanical/thermal hot stimuli and to inflammatory pain. We failed to observe OT receptor-mediated stress-induced analgesia as well as OT antihyperalgesia after carrageenan inflammation. These alterations were partially rescued if MS pups were treated by intraperitoneal daily injection during MS with OT or its downstream second messenger allopregnanolone. The involvement of epigenetic changes in these alterations was confirmed since neonatal treatment with the HDAC inhibitor SAHA, not only normalized nociceptive sensitivities, but also restored OT receptor-mediated stress-induced analgesia as well as the endogenous anti-hyperalgesia in inflamed MS rats. There is growing evidence in the literature that ELS might impair the nociceptive system ontogeny and function. This study suggests that these alterations might be restored while stimulating OT receptor signaling or HDAC inhibitors, using molecules that are currently available or part of clinical trials for other pathologies.
\end{abstract}


Pharmacological rescue of nociceptive hypersensitivity and oxytocin analgesia impairment in a rat model of neonatal maternal separation

Melchior $\mathrm{M}^{1}$, Juif $P E^{1}$, Gazzo $\mathrm{G}^{1}$, Petit-Demoulière $\mathrm{N}^{1}$, Chavant $\mathrm{V}^{1}$, Lacaud $\mathrm{A}^{1}$, Goumon $\mathrm{Y}^{1}$, Lelièvre $V^{1}$, Charlet $A^{1,2}$ \& Poisbeau $P^{1}$

1 Centre National de la Recherche Scientifique and University of Strasbourg, Institut des Neurosciences Cellulaires et Intégratives, F-67084 Strasbourg, France.

2 University of Strasbourg Institute for Advanced Study, Strasbourg, France

Corresponding author:

Prof. Pierrick Poisbeau, CNRS UPR-3212, Institut des Neurosciences Cellulaires et Intégratives

(INCI), Research group « Molecular Determinants of Pain », 5 rue Blaise Pascal, 67084 Strasbourg, France.

Tel: (+33) 368851476 - Fax: (+33) 388613347 - Email: poisbeau@inci-cnrs.unistra.fr 


\section{Pharmacological rescue of nociceptive hypersensitivity and oxytocin analgesia impairment in a rat model of neonatal maternal separation}

Melchior $\mathrm{M}^{1}$, Juif $\mathrm{PE}^{1}$, Gazzo $\mathrm{G}^{1}$, Petit-Demoulière $\mathrm{N}^{1}$, Chavant $\mathrm{V}^{1,2}$, Lacaud $\mathrm{A}^{1}$, Goumon $\mathrm{Y}^{1,2}$, Charlet $A^{1,3}$, Lelièvre $V^{1}, \&$ Poisbeau $P^{1}$

${ }^{1}$ Centre National de la Recherche Scientifique and University of Strasbourg, Institut des Neurosciences Cellulaires et Intégratives, F-67084 Strasbourg, France.

${ }^{2}$ Mass spectrometry facilities of the CNRS UPR3212, Institut des Neurosciences Cellulaires et Intégratives, Centre National de la recherche Scientifique, Strasbourg, France.

${ }^{3}$ University of Strasbourg Institute for Advanced Study, Strasbourg, France

Corresponding author:

Prof. Pierrick Poisbeau, CNRS UPR-3212, Institut des Neurosciences Cellulaires et Intégratives (INCl), Research group « Molecular Determinants of Pain », 5 rue Blaise Pascal, 67000 Strasbourg, France.

Tel: (+33) 368851476 - Fax: (+33) 388613347 - Email: poisbeau@inci-cnrs.unistra.fr 


\section{RESUME}

Oxytocin (OT), known for its neurohormonal effects around birth, has recently been suggested for being a critical determinant in neurodevelopmental disorders. This hypothalamic neuropeptide exerts a potent analgesic effect through an action on the nociceptive system. This endogenous control of pain has an important adaptive value but might be altered by early life stress (ELS), possibly contributing to its long-term consequences on pain responses and associated comorbidities. We tested this hypothesis using a rat model of neonatal maternal separation (NMS) known to induce long term consequences on several brain functions including chronic stress, anxiety, altered social behavior and visceral hypersensitivity. We found that adult rats with a history of NMS were hypersensitive to noxious mechanical/thermal hot stimuli and to inflammatory pain. We failed to observe OT receptor-mediated stress-induced analgesia as well as OT anti-hyperalgesia after carrageenan inflammation. These alterations were partially rescued if NMS pups were treated by intraperitoneal daily injection during NMS with OT or its downstream second messenger allopregnanolone. Involvement of epigenetic changes in these alterations was confirmed since neonatal treatment with the histone deacetylase (HDAC) inhibitor SAHA, not only normalized nociceptive sensitivities, but also restored OT receptor-mediated stress-induced analgesia as well as the endogenous anti-hyperalgesia in inflamed NMS rats. There is growing evidence in the literature that ELS might impair the nociceptive system ontogeny and function. This study suggests that these alterations might be restored while stimulating OT receptor signaling or HDAC inhibitors, using molecules that are currently available or part of clinical trials for other pathologies. 


\section{INTRODUCTION}

Oxytocin (OT) is a nonapeptide synthesized by hypothalamic neurons mainly located in the paraventricular (PVN) and supraoptic (SON) nuclei [1]. OT acts both as a neurohormone when released into the blood via the posterior pituitary or as a neurotransmitter when synaptically released into the spinal cord (SC) and supraspinal regions. Recently, our laboratory identified a small population of 30 parvocellular neurons in the PVN which coordinate the peripheral and spinal release of OT to limit inflammatory pain symptoms [2]. Indeed parvocellular OT neurons of the PVN trigger OT secretion in superficial and autonomic layers of the SC where OT receptors are found [1]. OT displays anti-nociceptive properties by increasing GABAergic inhibition in the SC thus decreasing excitability in superficial and deep SC neurons [1]. In addition to these central-related effects, a peripheral component of OT-mediated analgesia via a direct effect at the dorsal root ganglion (DRG) level has been documented [3].

Activation of the OT analgesic system occurs under different conditions such as inflammatory sensitization [2], neuropathic pain [4], or forced swim stress (FSS) [5]. In the carrageenan model of inflammatory sensitization, spinal OT levels are increased for at least $24 \mathrm{~h}$ and OT receptormediated local production of the neurosteroid allopregnanolone (AP) leads to a sustained GABAergic activity to maintain spinal antinociceptive control [6]. After a non-noxious FSS, OT is also responsible for significant and transient stress-induced analgesia (SIA) [5]. In this context, a deficit in OT signaling might be related to neurodevelopmental brain pathologies such as autism spectrum disorders $[7 ; 8]$.

The development of the nociceptive system starts before birth and follows a similar pattern in the rat and in the human fetus $[9 ; 10]$. It is well accepted that the developmental stage of the rat just after birth is similar to that of the human fetus at the beginning of the $3^{\text {rd }}$ trimester of gestation. 
Descending controls of pain mature during the first 3 weeks after birth in the rat [11] and begin to mature during the end of the $3^{\text {rd }}$ semester in humans [12]. At the SC level, spinal inputs and chloride homeostasis undergo re-organization steps after birth to be fully matured [13; 14] making them possibly vulnerable to early adverse events. Neonatal maternal separation (NMS) is a wellestablished model of early life stress (ELS) that induces long term neuroendocrine and behavioral changes in rodents, with impact on the HPA axis, social and cognitive behaviors, and sensory and nociceptive systems $[15 ; 16]$. Long-term consequences of NMS are indeed visceral hypersensitivity [17], epigenetic changes, and associated sensory dysfunctions [18; 19]. Recently, a chronic adult treatment with histone deacetylase (HDAC) inhibitor was shown to be efficient to counteract NMS-induced visceral hypersensitivity [19].

In this study, we used behavioral measures and in vivo electrophysiological recordings from SC dorsal horn neurons to characterize the long-term consequences of NMS on the oxytocinergic descending control of pain. Then, we aimed to prevent the development of NMS pain phenotype using pharmacological tools, targeting the OT system or epigenetic factors early during the postnatal period. 


\section{MATERIALS AND METHODS}

\section{Animal care and neonatal maternal deprivation procedure}

Female Wistar rats with litters were housed in a temperature-controlled room $\left(22^{\circ} \mathrm{C}\right)$ under a $12 \mathrm{~h}$ light-dark cycle (lights on at 07:00 AM), with ad libitum access to food and water. All experiments were conducted in conformity with the recommendations of the European Committee Council Direction of September 22, 2010 (2010/63/EU), with an authorization for animal experimentation from the French Department of Agriculture (License 67-116 to PP) and with a procedure agreement evaluated by the regional ethical committee (CREMEAS authorization number APAFIS\#7678-20 16112223027528 v2).

At birth, litters were randomly assigned to two groups: Neonatal NMS and non-separated control litters. The litters allocated to the NMS group were removed from the nest cages $3 \mathrm{~h}$ per day from postnatal days $2(\mathrm{P} 2)$ to $12(\mathrm{P} 12)$ and placed on a heating pad $\left(37^{\circ} \mathrm{C}\right)$ in a separate cage. The litters allocated to the control group remained with their mother in their home cages during the entire NMS interval and received no special handling other than that necessary to change the bedding of their cage. Pups were weaned at P21 and housed four rats per cage before being randomly chosen at 8 weeks or more for behavioral analysis or electrophysiological recordings. In each experiment, animals were chosen randomly in at least two different litters. Both males and females were used in this study, as no sex specific differences could be seen for the different neurophysiological and behavioral measures.

\section{Measure of basal mechanical and thermal nociception}

Mechanical nociception was measured on adult rats with a calibrated forceps (Bioseb, Vitrolles, France) as previously described [20]. Briefly, the habituated rat was loosely restrained with a towel 
masking the eyes in order to limit stress by environmental stimulations. The tips of the forceps were placed at each side of the paw and a gradually increasing force was applied. The pressure producing withdrawal of the paw corresponded to the nociceptive threshold value. Thermal nociception was measured by a blind experimenter with the Hargreaves method, as previously described [21]. Briefly, rats were placed in plexiglas boxes $(20 \mathrm{~cm} \times 25 \mathrm{~cm} \times 45 \mathrm{~cm})$ for a $15-20$ minutes habituation period and then hindpaws were exposed to a radiant heat and the latency time required to induce paw withdrawal was recorded and considered as the nociceptive heat threshold. For both tests, measurements were performed three times for each hindpaw and values were averaged.

\section{Forced swim stress}

At adulthood, rats were exposed to a forced swim stress procedure (FSS) in a Plexiglas ${ }^{\circledR}$ cylinder (diameter: $30 \mathrm{~cm}$; wall height: $60 \mathrm{~cm}$ ), filled with water (height: $40 \mathrm{~cm}$ ) at a temperature of $20^{\circ} \mathrm{C}$ for 10 minutes. After FSS exposure, animals were gently dried. Mechanical thresholds were measured before (baseline) and 15 min after FSS in both NMS and control animals. This protocol has been described to be associated with a plasmatic and spinal release of oxytocin [5]. The oxytocinergic contribution of FSS to SIA was confirmed in a subset of rats receiving an intrathecal (i.t) injection of $10 \mu \mathrm{L}$ of OTR antagonist dOVT $\left(\left(\mathrm{d}\left(\mathrm{CH}_{2}\right)_{5}{ }^{1}, \mathrm{Tyr}(\mathrm{Me})^{2}, \mathrm{Thr}^{4}, \mathrm{Orn}^{8}\right.\right.$, des-Gly- $\left.\mathrm{NH}_{2}{ }^{9}\right)$ -

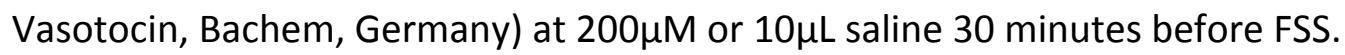

\section{Carrageenan Inflammatory pain model}

To induce a painful inflammatory sensitization in the rat, $150 \mu \mathrm{L}$ of carrageenan (3\% in $\mathrm{NaCl} 0.9 \%$, Sigma Aldrich, Germany) were injected in the plantar surface of the right hindpaw for behavioral 
experiments, whereas injections in both hindpaws were performed for electrophysiological. Control animals received either similar injections of $\mathrm{NaCl} 0.9 \%$ or no injection.

\section{In vivo recording of spinal cord neurons and analysis}

Male and female rats were anaesthetized with isoflurane ( $2 \%$; pushed by pressurized air at a flow rate of $0.5{\mathrm{~L} . \mathrm{min}^{-1}}^{-1}$ and body temperature was regulated using a thermostatically controlled heating blanket (Harvard Apparatus Ltd, France) maintaining the body temperature at $37^{\circ} \mathrm{C}$. Animals were set up in a stereotaxic frame (La Précision Cinématographique, Eaubonne, France), with the cervical and sacral vertebrae firmly held. The lumbar SC (L4-L5) was exposed by laminectomy. After removal of the meninges, the SC surface was covered with a thin isolating layer of mineral oil. This opening allowed us to record SC neurons and apply several drugs directly on the SC. At the end of the experiment, animals were killed with an overdose of isoflurane.

Single unit extracellular in vivo recordings were made from wide dynamic range (WDR) neurons, located in lamina $V$ and receiving peripheral sensory messages mediated by $A \beta, A \delta$ and C-type primary afferent fibers. In the lumbar enlargement of the SC, we monitored voltage changes through stainless microelectrodes (FHC, Bowdoin, USA) connected to an extracellular differential amplifier (DAM-80, WPI, Aston, UK). Electrical signals were then digitized (Power 1401 CED, Cambridge, UK), processed with the Spike 2 software (CED Cambridge, UK). Neurons were characterized by their responses to transcutaneous electrical stimulation of the hindpaw receptive field as previously described [22]. Electrical stimulation was applied through two thin cutaneous pin electrodes placed in the center of the receptive field on the hindpaw. Increasing intensities were applied from $1 \mathrm{~V}$ to $\mathrm{C}$ fiber activation threshold (duration of $1 \mathrm{~ms}$ ). Trials consisted of a train of 90 electrical stimuli ( $3 \times$ C-type fiber threshold) applied repetitively $(1 \mathrm{~Hz})$ to the excitatory 
receptive field. This method has been usually used to obtain a stable and reproducible "wind up" [23], a property of C-type nociceptive fibers. The electrically-evoked response of the neuron was captured and displayed as a post-stimulus histogram, for A-type and C-type discharge. Action potentials appearing early after the stimulus artifact were classified as A-type (0-50 ms) whereas the delayed response corresponded to C-type (50-800 ms).

\section{Spinal cord sampling and PCR analysis}

Lumbar spinal cord samples were collected, reconstituted in a guanidine thiocyanate/ $\beta$ mercaptoethanol preparation using ultraturax and stored at $-80^{\circ} \mathrm{C}$. Total RNA was extracted according to a protocol derived from the original procedure of Chomczynski and Sacchi [24], consisting of two independent total RNA extractions separated by a DNAsel treatment ((TURBO DNaseTM, Ambion, Life technologies, Saint Aubin, France), as previously described in detail [25]. 800ng RNA were used for reverse transcription with the RT iscript kit (Bio-Rad, Marnes-laCoquette, France). Quantitative PCR was performed using SYBR Green Supermix (Bio-Rad), on the iQ5 Real Time PCR System (Bio-Rad). Amplifications were carried out in 42 cycles (20s at 95 ${ }^{\circ} \mathrm{C}$, 20s at $60^{\circ} \mathrm{C}$ and $20 \mathrm{~s}$ at $72^{\circ} \mathrm{C}$ ). Standardization was made possible using standard curves made from serial dilutions of samples, and amplification data is shown as relative gene expression, calculated as the ratio between CDNA concentration of the gene of interest and that of the housekeeping gene. Preliminary experiments showed that among the different classic housekeeping genes routinely analyzed in the laboratory, and compared with $18 \mathrm{~S}$ ribosomal RNA, hypoxanthin-guanine phosphoribosyltransferase (HPRT) transcripts remained highly stable among the different samples. Therefore, HPRT was selected as the housekeeping gene to analyze and evaluate potential changes in gene expression in all experiments presented here. To specifically amplify mRNA encoding various rat proteins, we designed specific primer sets (sense and antisense 
respectively) using Oligo6.0 and M-fold softwares. Primer sequences for all genes of interest can

be found in supplementary table 1 . Samples were accurately dispensed in duplicates using a robotic workstation (Freedom EVO100, Tecan, Lyon, France), and amplification efficacy given by standard curves was always close to $100 \%( \pm 2 \%)$, whilst amplification specificity was assessed by a melting curve study.

\section{Neonatal rescue procedure}

To reverse NMS long term consequences on pain behavior, a pharmacological neonatal treatment was performed in NMS pups. During NMS protocol, all pups in the litter were injected with the same molecule and then tested at adulthood to investigate the long-term efficiency of neonatal treatment. OT (Bachem, Weil am Rhein, Germany), the HDAC inhibitor SAHA (Suberoylanilide hydroxamic acid, Vorinostat, ApexBio Technology, Houston, USA), or vehicle $(\mathrm{NaCl}$, oil or $10 \%$ DMSO respectively) were injected intraperitoneally (i.p. $10 \mu \mathrm{L}$ ) at $1 \mathrm{mg} / \mathrm{kg}$ and $5 \mathrm{mg} / \mathrm{kg}$ respectively once daily (o.d) during the NMS procedure (i.e from P2 to P12). In another subset of animals, allopregnanolone (AP, Santa Cruz biotechnology, Dallas, USA) was injected subcutaneously at $10 \mathrm{mg} / \mathrm{kg}$ once every two days during the NMS procedure. To avoid unnecessary supplementary handling of the pups, injections were performed at the beginning of the separation process. Pups were then normally weaned at P21, and no special handling was performed until P45, when the efficiency of neonatal treatment was assessed for each neonatal group using behavioral testing. First, mechanical thresholds were measured prior (baseline) and after FSS (SIA measurement). Then one week later, the efficacy of the OT descending control of pain after carrageenan inflammation of the hindpaw was measured with the same protocol as the one previously described (using spinal dOVT injection $24 \mathrm{H}$ after inflammation). 


\section{Drugs and Treatments}

OT receptor (OTR) agonists and antagonist, as well as $\mathrm{V} 1 \mathrm{aR}$ antagonist were prepared as a concentrated solution in water $(1 \mathrm{mM})$ and diluted to the needed concentration in $\mathrm{NaCl}(0.9 \%)$ before experiment. The selective OTR antagonist dOVT (d(CH2)5-Tyr(Me)-[Orn8]-vasotocine) was used at a final concentration of $1 \mu \mathrm{M}$ in Saline ( $\mathrm{NaCl} 0.9 \%$ ). dOVT was injected i.t. (volume $20 \mu \mathrm{L}$ ) or directly on the spinal cord in electrophysiological recordings (volume $500 \mu \mathrm{L})$. V1aR antagonist [Phenylacetyl1,O-Me-D-Tyr2,Arg6,8,Lys9]-vasopressinamide (AV1AR: Sigma Aldrich, Saint-Louis,

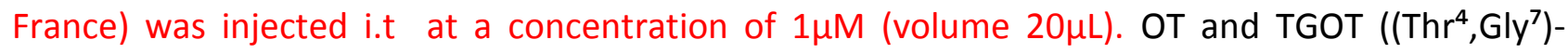
Oxytocin) (Bachem, Weil am Rhein, Germany) were used as an agonists for OTRs. OT was dissolved in saline and injected i.t or i.p. at a concentration of $10 \mu \mathrm{M}$ or $1 \mathrm{mg} / \mathrm{kg}$, respectively. TGOT was also dissolved in $\mathrm{NaCl} 0.9 \%$ and injected at a concentration of $1 \mu \mathrm{M}$ (i.t.). Allopregnanolone and SAHA were freshly prepared before the experiment. Allopregnanolone was used at $10 \mathrm{mg} / \mathrm{kg}$ and dissolved in mineral oil by sonication at $4^{\circ} \mathrm{C}$. The non-selective HDAC inhibitor SAHA was dissolved in $10 \%$ DMSO and injected i.p. at a concentration of $5 \mathrm{mg} / \mathrm{kg}$.

\section{$\underline{\text { Statistical analysis }}$}

All data are expressed as mean \pm standard error of the mean (SEM). Statistical analysis was performed using GraphPad Prism 6 software (LaJolla, CA, USA). For each experiment, parametric analysis was used only after verifying the normal distribution of the values. Unpaired Student ttest was used to compare baseline nociceptive sensitivity and OTR and oxytocinase (OTase) expression in NMS and CTRL rats. Repeated measures Two-Way ANOVA followed by Sidak post hoc test was used to analyze inflammatory symptoms and to analyze wind-up efficiency. Repeated measures Two-Way ANOVA followed by Tukey's post hoc test was used to evaluate the effect of 
OT and TGOT spinal application in control and NMS rats. One way ANOVA and Tukey post-hoc tests were used to compare the effects of spinal application of dOVT in control or NMS rats, to evaluate the efficiency of SIA in both groups, to analyze the success of the rescue experiments on baseline nociceptive sensitivities and to evaluate spinal mRNA expression of $3 \alpha$-hydroxysteroid

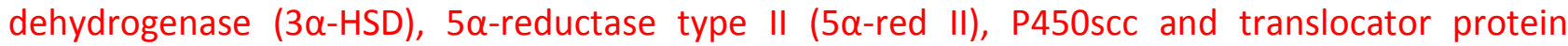
(TSPO). Mann-Whitney test was used to compare the effects of spinal application of the V1aR antagonist in control and NMS rats. Kruskal Wallis (KW) followed by Dunn's post hoc test was used to compare wind-up frequencies and spinal expression of histone deacetylases (HDAC) transcripts. Differences were considered to be statistically significant for $\mathrm{p}<0.05$. 


\section{RESULTS}

\section{Consequences of NMS on the nociceptive sensitivity and on the efficacy of oxytocinergic SIA}

We first characterized the long-term effects of NMS on baseline mechanical and thermal nociceptive thresholds (Figure 1A), as well as the intensity of stress-induced mechanical analgesia (SIA) measured after FSS (Figure 1B). Compared to the control group, NMS animals exhibited hypersensitivity to both mechanical and thermal stimulation at adulthood $(P>100)$. NMS rats displayed significantly lower mean pressure thresholds (control: $445.5 \pm 6.1 \mathrm{~g}, \mathrm{~N}=12$; NMS: 268.5 $\pm 7.1 \mathrm{~g}, \mathrm{~N}=12 ; \mathrm{p}<0.001$, unpaired Student $\mathrm{t}$-test, $\mathrm{t}=18.83, \mathrm{df}=22$ ) and paw withdrawal latencies to noxious heat (control: $21.1 \pm 0.8 \mathrm{~s}, \mathrm{~N}=12$; NMS: $12.6 \pm 0.4 \mathrm{~s}, \mathrm{~N}=12 ; \mathrm{p}<0.001$, unpaired Student t-test, $\mathrm{t}=9, \mathrm{df}=22$ ).

\section{${ }_{8}^{7}$ Figure 1 near here}

Previous studies showed that both central and peripheral release of OT contributed to SIA after a FSS [26; 22]. Because NMS is known to alter the normal reactivity to stress and lead to a dysfunction of the HPA axis, we investigated the efficacy of the oxytocinergic component of SIA in NMS animals (Figure 1B). As expected, the FSS paradigm induced a significant analgesia 20 minutes after swim in the control group (Figure 1B). Mean baseline-corrected mechanical thresholds were increased by $239 \pm 12.3 \mathrm{~g}$ in the control group $(\mathrm{N}=8)$ but only by $10.7 \pm 23.4 \mathrm{~g}$ in the NMS group ( $\mathrm{N}=8$ ). An injection of dOVT prior to FSS decreased SIA intensity to $56.9 \pm 20.1 \mathrm{~g}$ in the control group $(\mathrm{N}=8)$ and had no effect in the NMS group $(\mathrm{N}=8)$ where it varied of $20.75 \pm 20.7$ $g(p<0.001$, One-Way ANOVA, $F=28.9)$. These results suggest a blunted adaptation to stressful conditions in NMS rats associated with possible dysfunction of the oxytocinergic descending control of pain. 


\section{Behavioral response to a painful inflammatory sensitization by carrageenan}

We next compared the intensity and time course of inflammatory pain symptoms triggered by an intraplantar hindpaw injection of carrageenan to possibly highlight differences in the expression of mechanical/thermal hyperalgesia in control and NMS rats (Figure 2). In both groups, carrageenan injection induced a sustained mechanical and thermal hyperalgesia which peaked $7 \mathrm{~h}$ after injection, as previously described [6]. Although basal mechanical nociceptive thresholds (i.e., before carrageenan injection) were significantly lower in NMS rats, the time course of mechanical hyperalgesia was not significantly different between both groups (Figure 2A1-B1). In the control group, mean pressure threshold dropped from $445.8 \pm 18.7 \mathrm{~g}$ to $30.1 \pm 5.1 \mathrm{~g}, 7 \mathrm{~h}$ after injection $(N=6$; repeated measures two-way ANOVA, treatment $x$ time, $F(12,120)=63.81, p<0.001)$ and mechanical hyperalgesia lasted for at least 6 days for control. As mentioned above, at baseline, NMS rats exhibited a lower mean pressure threshold of $295.5 \pm 4.8 \mathrm{~g}(\mathrm{~N}=6)$, which further decreased to $70.8 \pm 12.6 \mathrm{~g}, 7 \mathrm{~h}$ after carrageenan injection. As illustrated in Figure 2B1, hyperalgesia was detected at a significant level for 6 days $(N=6$; repeated measures two-way ANOVA, treatment $x$ time, $\left.F_{(12,120)}=30.78, p<0.001\right)$. Concerning thermal hot sensitivity, $7 \mathrm{~h}$ after carrageenan injection mean latencies decreased to $3.3 \pm 0.6 \mathrm{~s}$ and $4.2 \pm 0.8 \mathrm{~s}$ in control and NMS rats ( $\mathrm{N}=6$ per group) respectively. Interestingly, thermal hot hyperalgesia persisted for at least 1 days for control rats and up to 4 days in NMS rats (Figure 2B2) (repeated measures two-way ANOVA, treatment $x$ time, $F_{(8,80)}=13.67, p<0.001$ for CTRL and $F_{(10,100)}=10.69, p<0.001$ for NMS).

${ }_{3}$ Figure 2 near here 
Oxytocinergic contribution to the intensity of carrageenan-induced pain symptoms and to the spinal cord processing of nociceptive messages

In the carrageenan model of inflammatory sensitization, spinal concentrations of OT are elevated during the early phase of inflammation and have been shown to limit pain sensitivity [22]. To investigate whether NMS affects OTergic anti-hyperalgesia, i.t application of the selective OTR antagonist dOVT was performed 24 hours after carrageenan injection. As OT may act via the activation of vasopressin V1a receptors (V1ARs) at high concentrations, we also examined a possible contribution of these receptors using i.t application of V1aR selective antagonist.

In the control group and as previously shown [6], a single i.t injection of dOVT $(1 \mu \mathrm{M}, 20 \mu \mathrm{L})$, induced a rebound of mechanical and thermal hyperalgesia. Anti-hyperalgesia mediated by OTR could be seen in the CTRL group as a decrease in the mean baseline-corrected mechanical threshold $4 \mathrm{~h}$ (i.e. $28 \mathrm{~h}$ after carrageenan) after dOVT injection by $-75.6 \pm 8.4 \mathrm{~g}(\mathrm{~N}=10$ Figure $3 \mathrm{~A})$. This decrease was absent in the saline-treated group $(-2 \pm 8.4 \mathrm{~g}, \mathrm{~N}=10)$ (One-way ANOVA $\mathrm{F}=17.5$, $p<0.001$ ). For thermal withdrawal latency (Figure 3B), OTR anti-hyperalgesia could also be seen as a significant decrease in the mean values after dOVT compared to saline ( $N=10$; One-way ANOVA, $F=9.3, p<0.001)$. This corresponded to a change of $-3.9 \pm 0.6 \mathrm{~s}(\mathrm{~N}=10)$ and of $1.1 \pm 0.8 \mathrm{~s}(\mathrm{~N}=10)$, for the dOVT and saline groups, respectively. Intrathecal injection of the V1aR antagonist was without effect on mechanical (before: $155.25 \pm 3.2 \mathrm{~g}$; after i.t. injection: $177.25 \pm 18.2 \mathrm{~g}, \mathrm{~N}=4$; Mann-Whitney test: $U=4.0 ; p=0.3429$ ) or thermal nociceptive threshold (before: $10.0 \pm 0.9 \mathrm{~s}$; after i.t. injection: $11.0 \pm 0.9 \mathrm{~s}, \mathrm{~N}=4$; Mann-Whitney test: $U=5.0 ; \mathrm{p}=0.4571$ ) in the control group of rats.

Both results confirmed OTR activation to be responsible for a limitation of pain symptoms early after carrageenan injection in control animals. Interestingly, there were no changes in the mean 


\section{igure 3 near here}

mechanical (dOVT: delta of $5.8 \pm 10.8 \mathrm{~g}, \mathrm{~N}=8$; saline: delta of $-27.2 \pm 9 \mathrm{~g}, \mathrm{~N}=7$; One-way ANOVA $F=17.5 p<0.001$ ) and thermal hyperalgesia values (dOVT: delta of $-1 \pm 0.7 \mathrm{~s}, \mathrm{~N}=8$; saline: delta of $0.1 \pm 0.7 \mathrm{~s}, \mathrm{~N}=7$; One-way ANOVA $\mathrm{F}=9.3 \mathrm{p}<0.001$ ) in NMS animals. As for control rats, a single i.t. injection of the V1aR antagonist did not change mechanical (before: $164.65 \pm 9.9 \mathrm{~g}$; after i.t. injection: $145.75 \pm 10.3 \mathrm{~g}, \mathrm{~N}=8$, Mann-Whitney test: $\mathrm{U}=20.0 ; \mathrm{p}=0.2241$ ) nor thermal nociceptive thresholds (before: $6.13 \pm 0.4$ s; after i.t. injection: $5.5 \pm 0.5 \mathrm{~s}, \mathrm{~N}=8$; Mann-Whitney test: $\mathrm{U}=22.0$; $p=0.3514)$ in NMS rat.

Together, this suggests that NMS has long-term consequences on the efficacy of OTR-mediated spinal analgesia, at least in short-term inflammatory painful sensitization.

To further characterize this NMS-altered OTR-mediated analgesia in NMS adult rats, we recorded WDR neurons in the SC of anesthetized rats and analyzed the spinal processing of nociceptive messages through the expression of windup, a nociceptive-specific short-term plasticity [27]. As illustrated in figure 4 , windup recorded $24 \mathrm{~h}$ after carrageenan injection was significantly amplified after the spinal application of dOVT $(1 \mu \mathrm{M})$. In control animals ( $N=6$, figure $4 \mathrm{~A})$, windup protocol led to an average maximum frequency of $23.6 \pm 1.5 \mathrm{spikes} / \mathrm{s}$. This value significantly increased to a maximum frequency of $44.1 \pm 4.8$ spikes/s after dOVT application $(N=6$; two-way repeated measures ANOVA, treatment $x$ time, $\left.F_{(14,70)}=7.811 ; p<0.0001\right)$. As shown in figure $4 B$, we did not see any change in windup frequencies in NMS animals $(\mathrm{N}=6)$ after dOVT application (before application: $26.8 \pm 4.3$ spikes/s; after application: $25 \pm 4.1$ spikes/s; two-way repeated measures ANOVA, treatment $x$ time, $\left.F_{(14,70)}=1.366 ; p=0.1936\right)$. Summary of results can be found in figure 4C (Kruskall-Wallis test: $\mathrm{KW}=8.9 ; \mathrm{p}=0.03)$. 
Altogether, electrophysiological and behavioral experiments suggested that the OT descending inhibitory control of pain is inefficient in NMS rats.

\section{OTR expression and OTR-induced analgesia}

In order to better understand the possible molecular and cellular alterations induced by NMS, we first analyzed the expression of OTR and a possible dysfunction of its downstream signaling, focusing on the SC as it is the first relay for nociceptive messages. There were no difference in the spinal expression of OTR gene transcripts between control $(\mathrm{N}=7 ; 3.1 \pm 0.9$ spinal relative expression) and NMS ( $N=8 ; 2.4 \pm 0.2$ spinal relative expression) rats (unpaired Student's t-test, $p=0.44, t=0.78, d f=13)$, as illustrated in figure $5 \mathrm{~A}$.

\section{${ }_{8}$ Figure 5 near here}

To further confirm that OTR were not only expressed at similar levels in both groups but were equally functional, we measured the analgesic behavioral response to i.t injection of OT $(1 \mu \mathrm{M}$ $20 \mu \mathrm{L})$ or to the selective agonist TGOT $(1 \mu \mathrm{M} 20 \mu \mathrm{L})$ in CTRL and NMS rats (Figure $5 \mathrm{~B})$. In CTRL animals OT $(\mathrm{N}=8)$ or TGOT $(\mathrm{N}=8)$ significantly increased mechanical thresholds 20 minutes after the injection (OT: from $467.4 \pm 19.4 \mathrm{~g}$ to $741.6 \pm 67.1 \mathrm{~g}$; TGOT: from $471.8 \pm 14.6 \mathrm{~g}$ to $813.2 \pm 26.3 \mathrm{~g} ; \quad$ Two-way repeated measures ANOVA, treatment $x$ time, $F_{(4,40)}=14.67$; $\mathrm{p}<0.0001)$. Both OT $(\mathrm{N}=7)$ or TGOT $(\mathrm{N}=8)$ significantly increased mechanical thresholds 20 minutes after the injection in a reversible manner in NMS animals (OT: from $288.4 \pm 5.0 \mathrm{~g}$ to $533.9 \pm 13.1$ g; TGOT: from $246.7 \pm 11.2$ g to $460.1 \pm 17.3$ g; Two-way repeated measures ANOVA, treatment $x$ time, $\left.F_{(11,38)}=66.94 ; p<0.001\right)$. This result confirmed that spinal OTR are functional in NMS rats and capable of producing efficient analgesia. We thus checked whether NMS could lead to an increase in the spinal degradation of OT. As illustrated in Supplementary Figure 1A, we found 
no difference in the spinal expression of oxytocinase (OTase) mRNA between control and NMS rats $(\mathrm{N}=8$; unpaired Student t-test, $\mathrm{t}=0.1317, \mathrm{df}=14 ; \mathrm{p}=0.8971)$.

\section{Neonatal treatments with OT and allopregnanolone}

Many studies provided evidence that OT in the neonatal period could be protective against early life adverse events or lesions [28; 29]. Moreover, NMS is known to reduce maternal behavior towards the pups and a subsequent altered development of OT systems, possibly leading to blunted OT levels [30]. This includes molecular mechanisms affecting chloride homeostasis and the production of neuroprotective neurosteroids such as allopregnanolone [6].

In a first set of experiments, we treated NMS rat pups o.d. from P2 to P12 with OT $(1 \mathrm{mg} / \mathrm{kg}$ in $0.9 \%$ $\mathrm{NaCl}$, i.p., Figure 6). As shown in figure $6 \mathrm{~A}$, neonatal OT treatment fully restored normal mechanical nociceptive thresholds when measured at P50 (control: $320.7 \pm 10.9 \mathrm{~g}, \mathrm{~N}=12$; NMS: $235.1 \pm 6.9 \mathrm{~g}, \quad \mathrm{~N}=8$; OT-treated NMS: $320.4 \pm 6.3 \mathrm{~g}, \quad \mathrm{~N}=10 ; \quad$ One-way ANOVA, $\mathrm{F}=23.39$, $\mathrm{p}<0.001$ ). OT neonatal treatment also restored an efficient analgesia after FSS (Figure 6B; delta for Control group: $261.8 \pm 18.6 \mathrm{~g}, \quad \mathrm{~N}=9$; NMS: $17.8 \pm 11.6 \mathrm{~g}, \quad \mathrm{~N}=8$; $\quad$ OT-treated NMS: $168.9 \pm 10.4 \mathrm{~g}, \mathrm{~N}=9$; One-way ANOVA, $\mathrm{F}=30.08, \mathrm{p}<0.001)$. In contrast to SIA after FSS, neonatal OT treatment failed to restore OT anti-hyperalgesia 24 hours after carrageenan-induced inflammation, measured as before using i.t dOVT injection (Figure 6C). The dOVT injection did not significantly amplified mechanical hyperalgesia (delta for NMS: $5.8 \pm 10.9 \mathrm{~g}, \mathrm{~N}=8$; OT-pretreated NMS: $45.6 \pm 19.7 \mathrm{~g}, \mathrm{~N}=8$; One-Way ANOVA, $\mathrm{F}=14.28, \mathrm{p}<0.001)$.

\section{Figure 6 near here}

Recently, an original long-lasting molecular mode of action of OT in SC neurons has been proposed. It leads to the stimulation of AP synthesis, a neurosteroid well-known for its analgesic 
and neuroprotective roles [31]. We thus tried to restore OT function in NMS rats by submitting newborn pups to AP during the NMS period. As illustrated in the different panels of figure 6 , a neonatal treatment with s.c AP $(10 \mathrm{mg} / \mathrm{kg})$ between $\mathrm{P} 2$ and $\mathrm{P} 12$ was associated with a mean mechanical threshold at adulthood which was higher (i.e., hyposensitivity) than those measured in control animals (Figure 6A; Control: $320.7 \pm 10.9 \mathrm{~g}, \mathrm{~N}=12$; AP-treated NMS: $394.1 \pm 21.9 \mathrm{~g}$, $N=10$; One way ANOVA, $F=23.39, p<0.001$ ). As for neonatal OT treatment, SIA could be restored by AP treatment (Figure 6B; baseline corrected mean mechanical threshold in AP-treated NMS: $\quad 351 \pm 47 \mathrm{~g}, \mathrm{~N}=8$, One Way ANOVA, $\mathrm{F}=30.08, \mathrm{p}<0.001$ ). As previously reported, endogenous OT anti-hyperalgesia could not be revealed after dOVT i.t. injection (Figure 6C; APtreated NMS: $-23 \pm 16 \mathrm{~g}, \mathrm{~N}=8$; One Way ANOVA, $\mathrm{F}=14.28, \mathrm{p}<0.001)$. Taken together, our results likely suggested NMS to be associated with a deficit in OT signaling. However, we found no significant difference in the spinal transcripts levels of $3 \alpha$-hydroxysteroid dehydrogenase $(3 \alpha-$

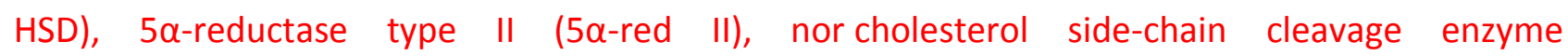
(P450scc), enzymes involved in AP synthesis (Supplementary Figure 1B; N = 8; One way ANOVA, $F=0.7409, p=0.6384$ ). Similarly, spinal translocator protein (TSPO) mRNA levels remained unchanged in NMS rats compared to controls.

NMS-induced epigenetic alterations and rescue of MS pain phenotype by targeting histone deacetylases

The long-term consequences of NMS on pain sensitivity and on the rat ability to cope with stress/pain potentially rely on epigenetic mechanisms. Indeed, several reports highlight the effects of NMS on the HPA axis and on maternal behavior [32; 33]. We thus measured the spinal expression of a set of transcripts coding for several epigenetic factors at different time points 
(from P7 to P100) in NMS and control animals (Figure 7A). We found that mRNA levels for some

class I (HDAC 1 and 8) and class II HDAC (HDAC 7) were significantly increased in NMS animals at P24 (HDAC 1 and 7), P45 (HDAC 1, 7 and 8) and until P100 for HDAC 7 (Kruskal-Wallis; HDAC1: $\mathrm{KW}=60.33, \mathrm{p}<0.0001, \mathrm{~N}=7 ; \mathrm{HDAC} 7: \mathrm{KW}=61.41, \mathrm{p}<0.0001, \mathrm{~N}=7 ; \mathrm{HDAC} 8: \mathrm{KW}=49.28$, $p<0.0001, N=6)$.

In particular, HDAC 7 mRNA spinal expression was significantly increased in NMS animals compared to controls at P24 (0.49 \pm 0.04 relative expression in CTRL and $1.22 \pm 0.11$ in NMS), as illustrated in figure 7B, and levels remained significantly higher until adulthood at P100 ( $0.36 \pm 0.03$ relative expression in CTRL and $1.01 \pm 0.04$ in NMS). These results likely suggest that NMS triggers long lasting changes in gene expression, involving at least HDAC functions.

\section{igure 7 near here}

We hence performed a neonatal chronic treatment from P2 to P12 with SAHA (10 mg/kg i.p.), a non-selective HDACI and II inhibitor. Neonatal treatment of newborn pups with SAHA during NMS was associated with mean mechanical nociceptive thresholds at adulthood (P50) which were similar to the control group (Figure 7C; Control: $320.7 \pm 10.9 \mathrm{~g}, \mathrm{~N}=8$; SAHA-treated NMS: $303.8 \pm 9.4 \mathrm{~g}, \mathrm{~N}=10$; One way ANOVA, $\mathrm{F}=14.28, \mathrm{p}<0.001)$. This treatment also partially restored SIA after FSS (Figure 7D; SAHA-treated: $130 \pm 26.7 \mathrm{~g}, \mathrm{n}=7$; One Way ANOVA, $\mathrm{F}=34.73, \mathrm{p}<0.001$ ). Interestingly, SAHA treatment partially restored OT anti-hyperalgesia after carrageenan inflammation (Figure 7E; Control: $75.6 \pm 8.4 \mathrm{~g}$; SAHA-treated: $-41.7 \pm 12.3 \mathrm{~g}$; One-Way ANOVA, $F=15.75, p<0.0001)$. 


\section{DISCUSSION}

In summary, we provide functional evidence indicating that NMS strongly affects adult nociception and the efficacy of the oxytocinergic descending inhibitory control. NMS is associated with (i) hypersensitivity to mechanical and thermal hot stimulation at adulthood, (ii) increased vulnerability to thermal inflammatory-induced hyperalgesia, and (iii) dysfunction of the OT descending control of pain after non-painful FSS or painful inflammatory sensitization. We further succeeded in rescuing these abnormalities in the nociceptive phenotype by repeated neonatal injections of OT, AP, or SAHA. Altogether, our data suggest that NMS has a strong impact on nociceptive integration, which can blunt the efficacy of descending controls of pain such as the oxytocinergic system which targets the spinal GABAergic inhibitory system. It also suggests that the OT or epigenetic systems can be good targets to develop early treatments to counteract the negative effects of ELS, at least in this model.

\section{Early life events promote nociceptive hypersensitivity and alter OTR-mediated analgesia}

Early life stress includes a wide range of adverse events which have long-term negative effects on brain function. In humans, ELS has been associated with an increased risk of developing chronic pain states at adulthood [34], including irritable bowel syndrome [35; 36]. In animal studies, the visceral hypersensitivity following NMS has been widely demonstrated, and correlated with differential activation of supraspinal regions, including the periaqueductal gray, which is a key area for descending pain controls. Here, we confirmed that nociceptive hypersensitivity extends to somatic mechanical and thermal hot stimuli, as previously published by our laboratory [16] and in other ELS models [37-40]. Our observation of an increased thermal pain symptom after carrageenan is consistent with studies showing an increased response after formalin injection [41] 
or after partial sciatic ligation [42] in NMS animals. Moreover, OT analgesic action on thermal sensitivity also relies on an action on TRPV1 receptors, since OT seems able to directly activate and promote the rapid desensitization of TRPV1 receptors expressed by C-type sensory fibers [43]. Although not tested in this study, we cannot exclude such an effect in NMS animals, as an increased duration of thermal hot hyperalgesia could be due to the loss of OT action on TRPV1 receptors.

Nociceptive hypersensitivity could be easily explained by a long-lasting up-regulation of voltagegated channels Nav1.8 and Nav1.9 in DRG neurons as recently published [16].

In this study, impairment of the oxytocinergic pain control has been revealed after recruitment by a non-painful or painful stress. The hypothalamus, where OT producing neurons are located, seems particularly vulnerable to ELS and a dysfunction of the oxytocinergic system may contribute to these alterations as OT plays a key role in the modulation of stress responses, anxiety, social interactions and pain responses. Despite several trials, we have not been able to demonstrate that endogenous OT concentrations are different between groups. Recently, NMS in rodents was shown to be associated with changes in OTR and V1aR binding sites in specific brain regions [44], suggesting a vulnerability of this system to the early environment. In humans, ELS has been associated with low oxytocin concentrations in CSF [45]. Here, we show that OT analgesia is impaired after NMS, both in painful and non-painful conditions. To exert its analgesic effects at the spinal level, OT relays on three main mechanisms: (i) activation of a spinal microcircuit leading to an increased GABAergic inhibitory tone [46], (ii) decrease of the excitability of SC neurons in the superficial layers [47] and (iii) stimulation of allopregnanolone synthesis which further amplifies GABAergic inhibition by allosteric modulation [6]. Here, we showed that spinal OTR are functional in NMS rats and that OTR activation can induce an efficient and transient analgesia, suggesting 
that the lack of OT-analgesia could hence result from supraspinal alterations. In agreement with our previously published results [22; 2], we failed to reveal any spinal contribution of the vasopressin V1A receptor in the fine tuning of basal nociceptive threshold or to induce oxytocinergic analgesia after painful or non-painful stress. This control was important since some discrepancies exist in the literature, often pointing out a specie-specific issue and a concentrationdependent action difficult to compare due to different administration procedures ([48]). Exploring hypothalamic OT neurons pharmacological and electrophysiological characteristics might help us to have a deeper understanding of the mechanisms leading to pain-related ELS consequences. A lack of AP synthesis following OTR activation could also explain the lack of OT anti-nociception following carrageenan inflammation in NMS rats, although we found no change in the spinal expression of transcripts coding for proteins responsible for AP synthesis in NMS rats, nor in the spinal expression of OTase mRNA.

\section{Rescuing NMS long-term alterations of the nociceptive system}

In our working hypothesis, we asked whether the lack of physical interaction between the mother and the pups could be a critical determinant to explain the deleterious plasticity of the nociceptive system and the deficit in oxytocinergic analgesia. Indeed, mother presence has already been shown to be protective against pain. Kangaroo mother care, in particular, is more frequently recommended and has been shown to have immediate analgesic effect on pain responses in the newborn $[49 ; 50]$ and to be protective against long term alterations usually following preterm birth [51; 52]. As OT has a key role in the modulation of parental behavior and attachment [53; 54], we performed a rescue experiment by repeated neonatal OT treatment during NMS, which reversed some consequences of NMS on pain behavior. In the literature, OT has been proposed as 
a therapeutic agent for different neurodevelopmental pathologies such as autism spectrum disorders, since it decreases anxiety and optimizes social behavior [7; 8]. In animal models, postnatal injections of OT have been shown to prevent feeding, social and memory deficit in the Prader-Willi model $[55 ; 29]$. Here, we only studied the beneficial consequences of an early treatment with OT on pain behaviors but further experiments would be helpful to confirm if this treatment could also counteract the detrimental effects of NMS on pain-related comorbidities such as stress and anxiety. These observations might have great clinical relevance in neonatal intensive care units since it confirms, once again, the crucial role of physical interactions between the parents and the newborn to optimize newborn development and install proper homeostatic controls to cope with stress (including pain).

A close reciprocal relationship exists between the oxytocinergic system and neurosteroids. Allopregnanolone is not only a downstream second messenger of OTR activation [6], it has wellknown anxiolytic, analgesic and neuroprotective properties [31]. In this study, a rescue experiment with neonatal allopregnanolone treatment appeared successful to restore normal pain sensitivity and a functional OT analgesia after FSS. Neonatal allopregnanolone level alterations can indeed lead to changes in behavior at adulthood [56]. Our result is also in good agreement with other studies using AP treatment to prevent neonatal anxiety after a short-term NMS (24 hours) [57]. The mechanisms by which AP could be protective against NMS consequences on pain controls are still unknown. However, neonatal AP level alterations could interact with chloride homeostasis development, since AP has also been shown to change KCC2 expression in the hippocampus [58]. Eventually, it is not surprising that HDAC inhibitor and epigenetic changes are involved in the longterm consequences of NMS on nociception and OT analgesia. It has long been known that neonatal stress has long lasting effects through epigenetic mechanisms [32; 59; 33]. Epigenetic 
changes in chromatin compaction through histone modification such as histone acetylation have

been demonstrated after NMS in different brain areas [59;60; 19]. Here we also demonstrate that the spinal expression of different pain-related epigenetic factors is altered after NMS, in particular HDAC 1, 7 and 8 which are overexpressed in a long-lasting manner compatible with the observed pain phenotypes. In the pain context, histone acetylation can modulate nociceptive response in inflammatory neuropathic and visceral pain models [61]. Spinal class II HDACs are involved in inflammatory hyperalgesia [62], which could explain the beneficial effects of SAHA treatment observed in this study after carrageenan inflammation, and the critical role of spinal HDAC 7 overexpression in NMS animals. In line, it has been suggested that suppressing HDAC 1 expression in the SC attenuated neuropathic pain symptoms [63]. The OTR gene promoter is also subjected to increased association of acH3K14 after NMS in rats, in critical structures for pain control [64]. However, we did not see any difference in the spinal expression of OTR in our study, providing further evidence that histone modification may be necessary but not sufficient to exert significant and long lasting changes in specific gene expression and that epigenetic alterations targeting the OTR gene may actually be region-dependant.

Corroborating our results, Moloney and colleagues showed that NMS is associated with an alteration in histone acetylation at the spinal level [19] and were the first to show that adult SAHA treatment can reverse NMS-induced visceral hypersensitivity. Here, we show that a preventive neonatal treatment with the same agent is also effective to prevent mechanical and thermal hypersensitivity at adulthood. At this stage, the underlying mechanisms are still to be identified as well as the molecular targets by which SAHA exerts its protective effects against NMS.

In conclusion, NMS induced both a basal hypersensitivity to pain and a dysfunction in OT descending control of pain, in painful and non-painful conditions. Some of these alterations can be 
rescued by neonatal SAHA or OT/AP treatment, but other alternatives need to be explored to be able to recover completely the OT antinociceptive action under inflammatory conditions. By studying the mechanisms of action of these pharmacological treatments, we might be able to identify new therapeutic targets to avoid NMS induced reprogramming of pain circuits.

\section{Acknowledgments}

This project has been supported by public funding from University of Strasbourg and CNRS. MM and GG are fellows of the French Ministry of research. PEJ was awarded a Gisele Guilbaud/Sfetd research price in 2013.

\section{Conflict of interest statement}

The authors have no conflict of interest. 


\section{FIGURES AND LEGENDS}

Figure 1. Altered baseline nociceptive sensitivity and stress induced analgesia efficacy in NMS rats. A. Baseline nociceptive thresholds for mechanical (left histogram) and thermal hot stimulation (right histogram) between control (black bars) and NMS rats (white bars). Statistical code for: Unpaired Student t-test ${ }^{* * *} p<0.001 \mathrm{~N}=12 /$ group. B. Stress induced-analgesia (SIA) detected 20 minutes after a forced swim stress procedure aimed at stimulating OT endogenous controls ( 10 minutes, water at $20^{\circ} \mathrm{C}$ ) for control ( $\mathrm{N}=8$ black bar), NMS ( $\mathrm{N}=8$ white bar), control preinjected with dOVT ( $\mathrm{N}=8$ red bar) and NMS rats pre-injected with dOVT ( $\mathrm{N}=8$ grey bar). The variation of mechanical threshold in grams has been calculated as: Delta $=$ mechanical threshold post FSS - mechanical threshold pre-FSS. Statistical code for One Way ANOVA followed by Tukey post hoc test: ${ }^{* * *} p<0.001$.

Figure 2. Increased sensibility to inflammatory pain in NMS rats. Time course of mechanical (top panels) and thermal hot (bottom panels) nociceptive thresholds for control (A) and NMS rats (B). Carrageenan injection was done at day 0 just after the daily measure. Note that the duration of thermal hyperalgesia was longer for NMS rats. Statistical code for repeated measures Two Way ANOVA treatment $\mathrm{X}$ time followed by Sidak post hoc test to compare ipsilateral and contralateral hindpaws for each time point: ${ }^{* *} \mathrm{p}<0.01,{ }^{* * *} \mathrm{p}<0.001 \mathrm{~N}=12$ per group.

Figure 3. Lack of efficiency of OT anti-hyperalgesic control on behavioral symptoms of inflammatory pain in NMS rats. Changes in the mean mechanical (A) and thermal hot (B) thresholds, $24 \mathrm{~h}$ after a carrageenan hindpaw injection, in control $(\mathrm{N}=10)$ and NMS rats $(\mathrm{N}=8)$ submitted to a single injection of the selective OTR antagonist dOVT $(1 \mu \mathrm{M}, 20 \mu \mathrm{L})$ or saline ( $\mathrm{NaCl}$ $0.9 \%, 20 \mu \mathrm{L})$, measured as: Delta $=$ threshold post dOVT injection- threshold pre dOVT injection . 
Statistical code for One Way ANOVA and Tukey post hoc test: ${ }^{*} p<0.05,{ }^{* *} p<0.01, * * * p<0.001$. CTRL: control, NMS: maternal separation, s: saline.

Figure 4. Lack of efficiency of OT anti-nociceptive control on spinal cord wide dynamic range neurons. A and B. Number of action potentials measured during windup when recording from wide dynamic range neurons in vivo. Results are given for anesthetized control (CTRL, A) and NMS rats (B), displaying an hindpaw inflammation for $24 \mathrm{~h}$ due to carrageenan injection, and submitted to a repetitive stimulation of the corresponding receptive field on the hindpaw $(1 \mathrm{~Hz}$, intensity: $3 x$ threshold for $\mathrm{C}$ fibers). Windup characteristics are provided before (black symbols) and 20 minutes after local (spinal) application of the selective antagonist dOVT $(1 \mu \mathrm{M}$, in red). Statistical code for repeated measures Two Way ANOVA Treatment $X$ Time and Sidak post hoc test: ${ }^{*} p<0.05$, ${ }^{* * *} p<0.001$. C. Mean frequencies of action potential discharge at wind-up plateau phase for all groups. Statistical code for Kruskal Wallis test and Sidak post hoc test: ${ }^{*} p<0.05 \mathrm{~N}=6$ per group.

Figure 5. Function of OTR in NMS rats. A. Expression of transcripts coding for spinal OTR in control and NMS rats. No significant difference between groups has been detected using Unpaired Student t-test, $\mathrm{N}=7$ control and $\mathrm{N}=8 \mathrm{NMS}$. B. Changes in mechanical nociceptive threshold in $\mathrm{CTRL}$ and NMS rats after i.t injection of OT ( $1 \mu \mathrm{M}, 20 \mu \mathrm{L}$, green triangles, $\mathrm{N}=8$ for CTRL and $\mathrm{N}=7$ for NMS), TGOT $(1 \mu \mathrm{M}, 20 \mu \mathrm{L}$, orange squares, $\mathrm{N}=8)$ or vehicle $(\mathrm{NaCl}, 20 \mu \mathrm{L}$, white circles, $\mathrm{N}=7)$. Statistical code for repeated measures Two-way ANOVA treatment $X$ Time followed by Tukey's post hoc test: $* * * p<0.001$.

Figure 6. Neonatal treatment with OT or AP can partially suppress NMS consequences. Effect of a neonatal repeated treatment with oxytocin (OT, $1 \mathrm{mg} / \mathrm{kg}$ o.d. i.p. injections between P2 and P12, purple bars) or allopregnanolone (AP, $10 \mathrm{mg} / \mathrm{kg}$ i.p. injections between P2 and P12 every 2days, red bars), on mechanical nociceptive thresholds $(A, N=12$ control and $N=8 N M S)$, stress induced 
analgesia (SIA) (B, N=9 control and $\mathrm{N}=8 \mathrm{NMS})$, and OTR-dependent inhibitory control $24 \mathrm{~h}$ after carrageenan inflammation (C, $\mathrm{n}=8$ per group). Results are shown for control (CTRL) and NMS rats. Statistical code for One WAY ANOVA followed by Tukey post hoc test: ${ }^{*} p<0.05 ;{ }^{* *} p<0.01$; $* * * p<0.001$.

Figure 7. Epigenetic alterations following NMS and partial suppression of NMS consequences after a neonatal treatment with SAHA. A. Changes in relative gene expression of spinal transcripts coding for HDAC 1-9 in NMS rats compared to controls, as measured with RT-qPCR, from P7 to P100. A significant increase in the expression of HDAC 1, HDAC 7 and HDAC 8 has been detected at different time points. Statistical code for Kruskal-Wallis followed by Dunn's post hoc test : $++p<0.01 ;+++p<0.001$, with " + " symbolizing and increased in expression in NMS rats compared to controls. B. Time course of the expression of transcripts coding for spinal HDAC 7 in control and NMS rats, from P7 to P100. Statistical code for Kruskal-Wallis followed by Dunn's post hoc test : ${ }^{* *} \mathrm{p}<0.01 ;{ }^{* *} \mathrm{p}<0.001$. C to E. Effect of a neonatal repeated treatment with SAHA (5mg/kg, daily i.p. injection between P2 and P12: dark green), a non-selective HDAC inhibitor, on mechanical nociceptive thresholds (C, N=8 control and NMS, N=10 SAHA treated NMS), stress-induced analgesia ( $D, N=9$ CTRL, $N=8$ NMS and $N=7$ SAHA treated NMS), OTRdependent inhibitory control 24 hours after carrageenan inflammation $(E, N=10$ ctrl, N=8 NMS N=8 SAHA treated NMS). Results are shown for control (CTRL) and NMS rats. Statistical code for One WAY ANOVA followed by Tukey post hoc test for panels $C$ to $E:{ }^{*} p<0.05 ;{ }^{* * *} p<0.001$.

Supplementary Figure 1. A. Expression of transcripts coding for spinal OTase in control and NMS rats, as measured by RT-qPCR. No significant difference between conditions has been detected using unpaired Student t-test, $\mathrm{N}=8 \mathrm{CTRL}$ and NMS. B. Expression of transcripts coding for spinal 3 $\alpha$-HSD, 5 $\alpha$-reductase II, P450scc and TSPO in control and NMS rats, as measured by RT- 
qPCR. No significant difference between conditions has been detected using One Way ANOVA, N=8 CTRL and NMS.

Supplementary Table 1. List of primer sequences used to amplify genes of interest by quantitative RT-PCR. 


\section{REFERENCES}

[1]Poisbeau P, Grinevich V, Charlet A. Oxytocin Signaling in Pain: Cellular, Circuit, System, and Behavioral Levels. Curr Top Behav Neurosci 2017.

[2]Eliava M, Melchior M, Knobloch-Bollmann HS, Wahis J, da Silva Gouveia M, Tang Y, Ciobanu AC, Triana Del Rio R, Roth LC, Althammer F, Chavant V, Goumon Y, Gruber T, PetitDemouliere N, Busnelli M, Chini B, Tan LL, Mitre M, Froemke RC, Chao MV, Giese G, Sprengel R, Kuner R, Poisbeau P, Seeburg PH, Stoop R, Charlet A, Grinevich V. A New Population of Parvocellular Oxytocin Neurons Controlling Magnocellular Neuron Activity and Inflammatory Pain Processing. Neuron 2016;89(6):1291-1304.

[3]Moreno-Lopez Y, Martinez-Lorenzana G, Condes-Lara M, Rojas-Piloni G. Identification of oxytocin receptor in the dorsal horn and nociceptive dorsal root ganglion neurons. Neuropeptides 2013;47(2):117-123.

[4]Martinez-Lorenzana G, Espinosa-Lopez L, Carranza M, Aramburo C, Paz-Tres C, Rojas-Piloni G, Condes-Lara M. PVN electrical stimulation prolongs withdrawal latencies and releases oxytocin in cerebrospinal fluid, plasma, and spinal cord tissue in intact and neuropathic rats. Pain 2008;140(2):265-273.

[5]Wotjak CT, Ganster J, Kohl G, Holsboer F, Landgraf R, Engelmann M. Dissociated central and peripheral release of vasopressin, but not oxytocin, in response to repeated swim stress: new insights into the secretory capacities of peptidergic neurons. Neuroscience 1998;85(4):12091222 .

[6]Juif PE, Breton JD, Rajalu M, Charlet A, Goumon Y, Poisbeau P. Long-lasting spinal oxytocin analgesia is ensured by the stimulation of allopregnanolone synthesis which potentiates GABA(A) receptor-mediated synaptic inhibition. J Neurosci 2013;33(42):16617-16626.

[7]Modi ME, Young LJ. The oxytocin system in drug discovery for autism: animal models and novel therapeutic strategies. Horm Behav 2012;61(3):340-350.

[8]Young LJ, Barrett CE. Neuroscience. Can oxytocin treat autism? Science 2015;347(6224):825826.

[9]Simons SH, Tibboel D. Pain perception development and maturation. Semin Fetal Neonatal Med 2006;11(4):227-231.

[10]Schwaller F, Fitzgerald M. The consequences of pain in early life: injury-induced plasticity in developing pain pathways. Eur J Neurosci 2014;39(3):344-352.

[11]Fitzgerald M. The development of nociceptive circuits. Nat Rev Neurosci 2005;6(7):507-520.

[12]Hartley C, Moultrie F, Gursul D, Hoskin A, Adams E, Rogers R, Slater R. Changing Balance of Spinal Cord Excitability and Nociceptive Brain Activity in Early Human Development. Curr Biol 2016;26(15):1998-2002. 
[13]Beggs S, Torsney C, Drew LJ, Fitzgerald M. The postnatal reorganization of primary afferent input and dorsal horn cell receptive fields in the rat spinal cord is an activity-dependent process. Eur J Neurosci 2002;16(7):1249-1258.

[14]Ben-Ari Y. The GABA excitatory/inhibitory developmental sequence: a personal journey. Neuroscience 2014;279:187-219.

[15]O'Mahony SM, Hyland NP, Dinan TG, Cryan JF. Maternal separation as a model of brain-gut axis dysfunction. Psychopharmacology (Berl) 2011;214(1):71-88.

[16]Juif PE, Salio C, Zell V, Melchior M, Lacaud A, Petit-Demouliere N, Ferrini F, Darbon P, Hanesch U, Anton F, Merighi A, Lelievre V, Poisbeau P. Peripheral and central alterations affecting spinal nociceptive processing and pain at adulthood in rats exposed to neonatal maternal deprivation. Eur J Neurosci 2016;44(3):1952-1962.

[17]Sengupta JN. Visceral pain: the neurophysiological mechanism. Handb Exp Pharmacol 2009(194):31-74.

[18]Theodorou V. Susceptibility to stress-induced visceral sensitivity: a bad legacy for next generations. Neurogastroenterol Motil 2013;25(12):927-930.

[19]Moloney RD, Stilling RM, Dinan TG, Cryan JF. Early-life stress-induced visceral hypersensitivity and anxiety behavior is reversed by histone deacetylase inhibition. Neurogastroenterol Motil 2015;27(12):1831-1836.

[20]Luis-Delgado OE, Barrot M, Rodeau JL, Schott G, Benbouzid M, Poisbeau P, Freund-Mercier MJ, Lasbennes F. Calibrated forceps: a sensitive and reliable tool for pain and analgesia studies. J Pain 2006;7(1):32-39.

[21]Hargreaves K, Dubner R, Brown F, Flores C, Joris J. A new and sensitive method for measuring thermal nociception in cutaneous hyperalgesia. Pain 1988;32(1):77-88.

[22]Juif PE, Poisbeau P. Neurohormonal effects of oxytocin and vasopressin receptor agonists on spinal pain processing in male rats. Pain 2013;154(8):1449-1456.

[23]Mendell LM. Physiological properties of unmyelinated fiber projection to the spinal cord. Exp Neurol 1966;16(3):316-332.

[24]Chomczynski P, Sacchi N. Single-step method of RNA isolation by acid guanidinium thiocyanate-phenol-chloroform extraction. Anal Biochem 1987;162(1):156-159.

[25]Lelievre V, Hu Z, Byun JY, Ioffe Y, Waschek JA. Fibroblast growth factor-2 converts PACAP growth action on embryonic hindbrain precursors from stimulation to inhibition. J Neurosci Res 2002;67(5):566-573.

[26]Robinson DA, Wei F, Wang GD, Li P, Kim SJ, Vogt SK, Muglia LJ, Zhuo M. Oxytocin mediates stress-induced analgesia in adult mice. J Physiol 2002;540(Pt 2):593-606.

[27]Herrero JF, Laird JM, Lopez-Garcia JA. Wind-up of spinal cord neurones and pain sensation: much ado about something? Prog Neurobiol 2000;61(2):169-203. 
[28]Bakos J, Lestanova Z, Strbak V, Havranek T, Bacova Z. Neonatal manipulation of oxytocin prevents lipopolysaccharide-induced decrease in gene expression of growth factors in two developmental stages of the female rat. Neuropeptides 2014;48(5):281-286.

[29]Meziane H, Schaller F, Bauer S, Villard C, Matarazzo V, Riet F, Guillon G, Lafitte D, Desarmenien MG, Tauber M, Muscatelli F. An Early Postnatal Oxytocin Treatment Prevents Social and Learning Deficits in Adult Mice Deficient for Magel2, a Gene Involved in Prader-Willi Syndrome and Autism. Biol Psychiatry 2015;78(2):85-94.

[30]Lovic V, Gonzalez A, Fleming AS. Maternally separated rats show deficits in maternal care in adulthood. Dev Psychobiol 2001;39(1):19-33.

[31]Poisbeau P, Keller AF, Aouad M, Kamoun N, Groyer G, Schumacher M. Analgesic strategies aimed at stimulating the endogenous production of allopregnanolone. Front Cell Neurosci $2014 ; 8: 174$.

[32]Champagne FA. Epigenetic mechanisms and the transgenerational effects of maternal care. Front Neuroendocrinol 2008;29(3):386-397.

[33]Buschdorf JP, Meaney MJ. Epigenetics/Programming in the HPA Axis. Compr Physiol 2015;6(1):87-110.

[34]Davis DA, Luecken LJ, Zautra AJ. Are reports of childhood abuse related to the experience of chronic pain in adulthood? A meta-analytic review of the literature. Clin J Pain 2005;21(5):398-405.

[35]Salmon P, Skaife K, Rhodes J. Abuse, dissociation, and somatization in irritable bowel syndrome: towards an explanatory model. J Behav Med 2003;26(1):1-18.

[36]Ross CA. Childhood sexual abuse and psychosomatic symptoms in irritable bowel syndrome. J Child Sex Abus 2005;14(1):27-38.

[37]Takatsuru Y, Yoshitomo M, Nemoto T, Eto K, Nabekura J. Maternal separation decreases the stability of mushroom spines in adult mice somatosensory cortex. Brain Res 2009;1294:4551 .

[38]Pierce AN, Ryals JM, Wang R, Christianson JA. Vaginal hypersensitivity and hypothalamicpituitary-adrenal axis dysfunction as a result of neonatal maternal separation in female mice. Neuroscience 2014;263:216-230.

[39]Yasuda M, Shinoda M, Honda K, Fujita M, Kawata A, Nagashima H, Watanabe M, Shoji N, Takahashi O, Kimoto S, Iwata K. Maternal Separation Induces Orofacial Mechanical Allodynia in Adulthood. J Dent Res 2016;95(10):1191-1197.

[40]Amini-Khoei H, Amiri S, Mohammadi-Asl A, Alijanpour S, Poursaman S, Haj-Mirzaian A, Rastegar M, Mesdaghinia A, Banafshe HR, Sadeghi E, Samiei E, Mehr SE, Dehpour AR. Experiencing neonatal maternal separation increased pain sensitivity in adult male mice: Involvement of oxytocinergic system. Neuropeptides 2017;61:77-85. 
[41]Uhelski ML, Fuchs PN. Maternal separation stress leads to enhanced emotional responses to noxious stimuli in adult rats. Behav Brain Res 2010;212(2):208-212.

[42]Nishinaka T, Kinoshita M, Nakamoto K, Tokuyama S. Sex differences in depression-like behavior after nerve injury are associated with differential changes in brain-derived neurotrophic factor levels in mice subjected to early life stress. Neurosci Lett 2015;592:3236 .

[43]Nersesyan Y, Demirkhanyan L, Cabezas-Bratesco D, Oakes V, Kusuda R, Dawson T, Sun X, Cao C, Cohen AM, Chelluboina B, Veeravalli KK, Zimmermann K, Domene C, Brauchi S, Zakharian E. Oxytocin Modulates Nociception as an Agonist of Pain-Sensing TRPV1. Cell Rep 2017;21(6):1681-1691.

[44]Lukas M, Bredewold R, Neumann ID, Veenema AH. Maternal separation interferes with developmental changes in brain vasopressin and oxytocin receptor binding in male rats. Neuropharmacology 2010;58(1):78-87.

[45]Heim C, Young LJ, Newport DJ, Mletzko T, Miller AH, Nemeroff CB. Lower CSF oxytocin concentrations in women with a history of childhood abuse. Mol Psychiatry 2009;14(10):954-958.

[46]Breton JD, Veinante P, Uhl-Bronner S, Vergnano AM, Freund-Mercier MJ, Schlichter R, Poisbeau P. Oxytocin-induced antinociception in the spinal cord is mediated by a subpopulation of glutamatergic neurons in lamina I-II which amplify GABAergic inhibition. Mol Pain 2008;4:19.

[47]Breton JD, Poisbeau P, Darbon P. Antinociceptive action of oxytocin involves inhibition of potassium channel currents in lamina II neurons of the rat spinal cord. Mol Pain 2009;5:63.

[48]Schorscher-Petcu A, Sotocinal S, Ciura S, Dupre A, Ritchie J, Sorge RE, Crawley JN, Hu SB, Nishimori K, Young LJ, Tribollet E, Quirion R, Mogil JS. Oxytocin-induced analgesia and scratching are mediated by the vasopressin-1A receptor in the mouse. J Neurosci 2010;30(24):8274-8284.

[49]Johnston CC, Stevens B, Pinelli J, Gibbins S, Filion F, Jack A, Steele S, Boyer K, Veilleux A. Kangaroo care is effective in diminishing pain response in preterm neonates. Arch Pediatr Adolesc Med 2003;157(11):1084-1088.

[50]Ludington-Hoe SM, Hosseini R, Torowicz DL. Skin-to-skin contact (Kangaroo Care) analgesia for preterm infant heel stick. AACN Clin Issues 2005;16(3):373-387.

[51]Feldman R, Rosenthal Z, Eidelman AI. Maternal-preterm skin-to-skin contact enhances child physiologic organization and cognitive control across the first 10 years of life. Biol Psychiatry 2014;75(1):56-64.

[52]Charpak N, Tessier R, Ruiz JG, Hernandez JT, Uriza F, Villegas J, Nadeau L, Mercier C, Maheu F, Marin J, Cortes D, Gallego JM, Maldonado D. Twenty-year Follow-up of Kangaroo Mother Care Versus Traditional Care. Pediatrics 2017;139(1). 
[53]Carter CS. Oxytocin pathways and the evolution of human behavior. Annu Rev Psychol 2014;65:17-39.

[54]Feldman R. The Neurobiology of Human Attachments. Trends Cogn Sci 2017;21(2):80-99.

[55]Schaller F, Watrin F, Sturny R, Massacrier A, Szepetowski P, Muscatelli F. A single postnatal injection of oxytocin rescues the lethal feeding behaviour in mouse newborns deficient for the imprinted Magel2 gene. Hum Mol Genet 2010;19(24):4895-4905.

[56]Darbra S, Modol L, Llido A, Casas C, Vallee M, Pallares M. Neonatal allopregnanolone levels alteration: effects on behavior and role of the hippocampus. Prog Neurobiol 2014;113:95105 .

[57]Llido A, Modol L, Darbra S, Pallares M. Interaction between neonatal allopregnanolone administration and early maternal separation: effects on adolescent and adult behaviors in male rat. Horm Behav 2013;63(4):577-585.

[58]Modol L, Casas C, Llido A, Navarro X, Pallares M, Darbra S. Neonatal allopregnanolone or finasteride administration modifies hippocampal $\mathrm{K}(+) \mathrm{Cl}(-)$ co-transporter expression during early development in male rats. J Steroid Biochem Mol Biol 2014;143:343-347.

[59]Murgatroyd C, Spengler D. Epigenetic programming of the HPA axis: early life decides. Stress 2011;14(6):581-589.

[60]Levine A, Worrell TR, Zimnisky R, Schmauss C. Early life stress triggers sustained changes in histone deacetylase expression and histone $\mathrm{H} 4$ modifications that alter responsiveness to adolescent antidepressant treatment. Neurobiol Dis 2012;45(1):488-498.

[61]Wang W, Cui SS, Lu R, Zhang H. Is there any therapeutic value for the use of histone deacetylase inhibitors for chronic pain? Brain Res Bull 2016;125:44-52.

[62]Bai G, Wei D, Zou S, Ren K, Dubner R. Inhibition of class II histone deacetylases in the spinal cord attenuates inflammatory hyperalgesia. Mol Pain 2010;6:51.

[63]Cherng CH, Lee KC, Chien CC, Chou KY, Cheng YC, Hsin ST, Lee SO, Shen CH, Tsai RY, Wong CS. Baicalin ameliorates neuropathic pain by suppressing HDAC1 expression in the spinal cord of spinal nerve ligation rats. J Formos Med Assoc 2014;113(8):513-520.

[64]Litvin Y, Turner CA, Rios MB, Maras PM, Chaudhury S, Baker MR, Blandino P, Jr., Watson SJ, Jr., Akil H, McEwen B. Fibroblast growth factor 2 alters the oxytocin receptor in a developmental model of anxiety-like behavior in male rat pups. Horm Behav 2016;86:6470 . 
Maternal separation renders adult rats hypersensitive to noxious stimuli and incapable to recruit oxytocin analgesic controls. Rescue is achieved by neonatal treatments with oxytocin, allopregnanolone or HDAC inhibitors. 
A.

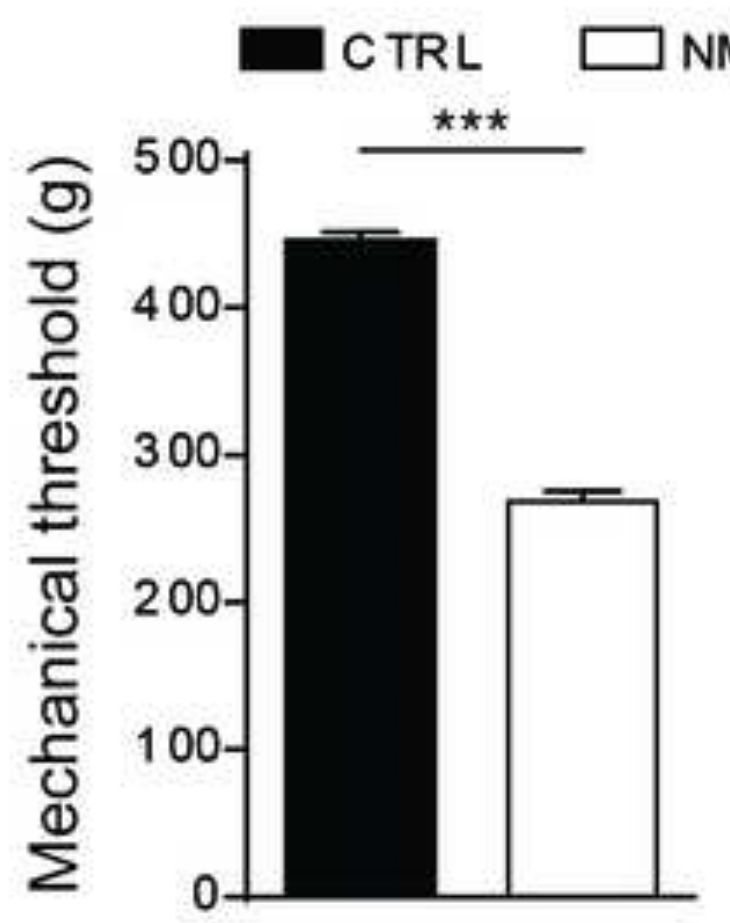

B.

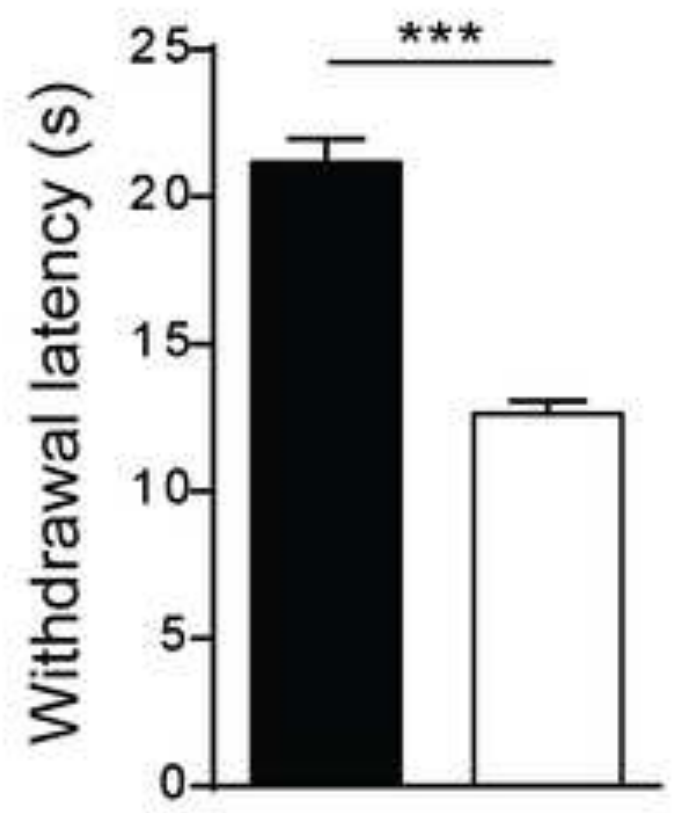

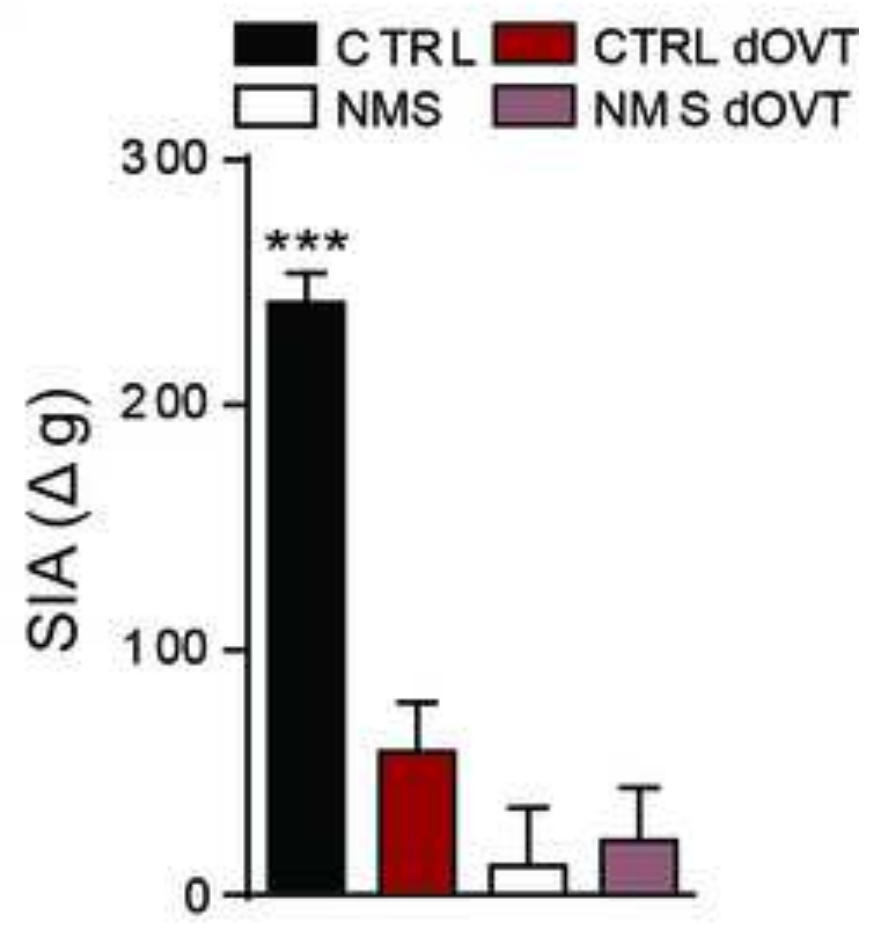




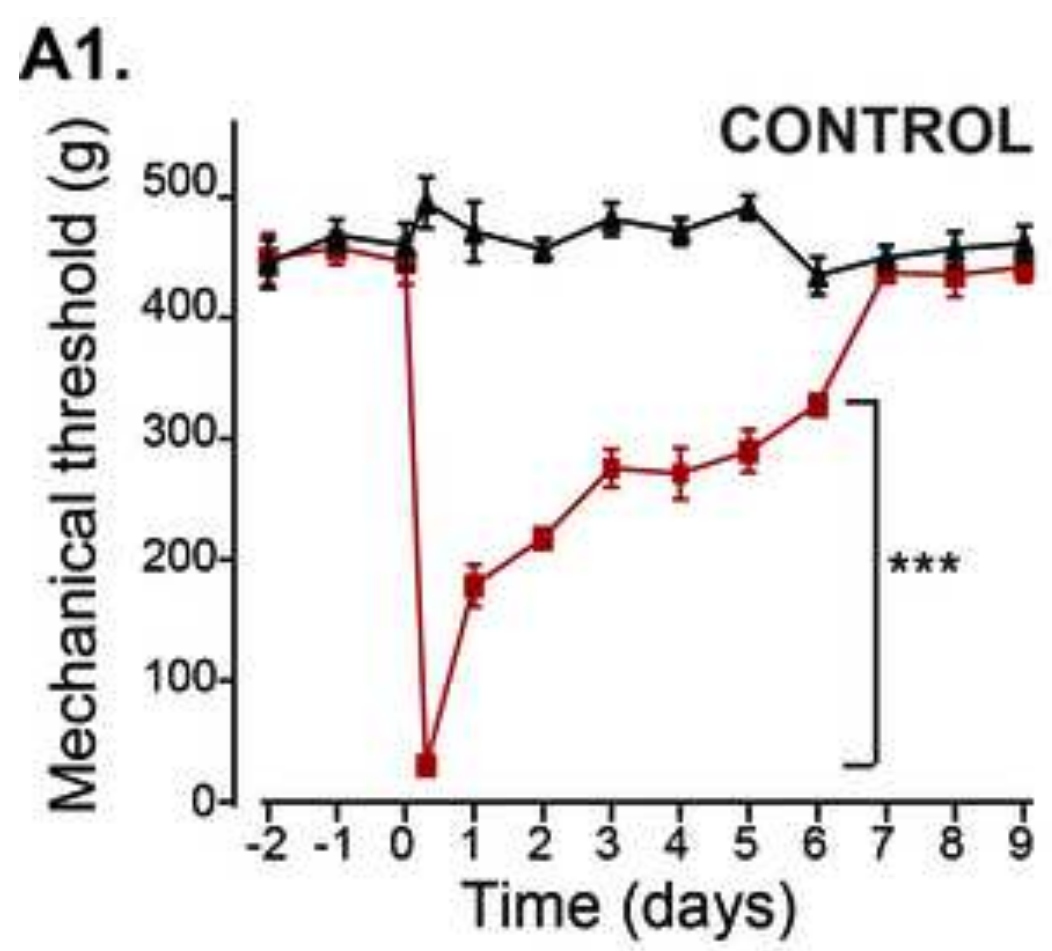

B1.

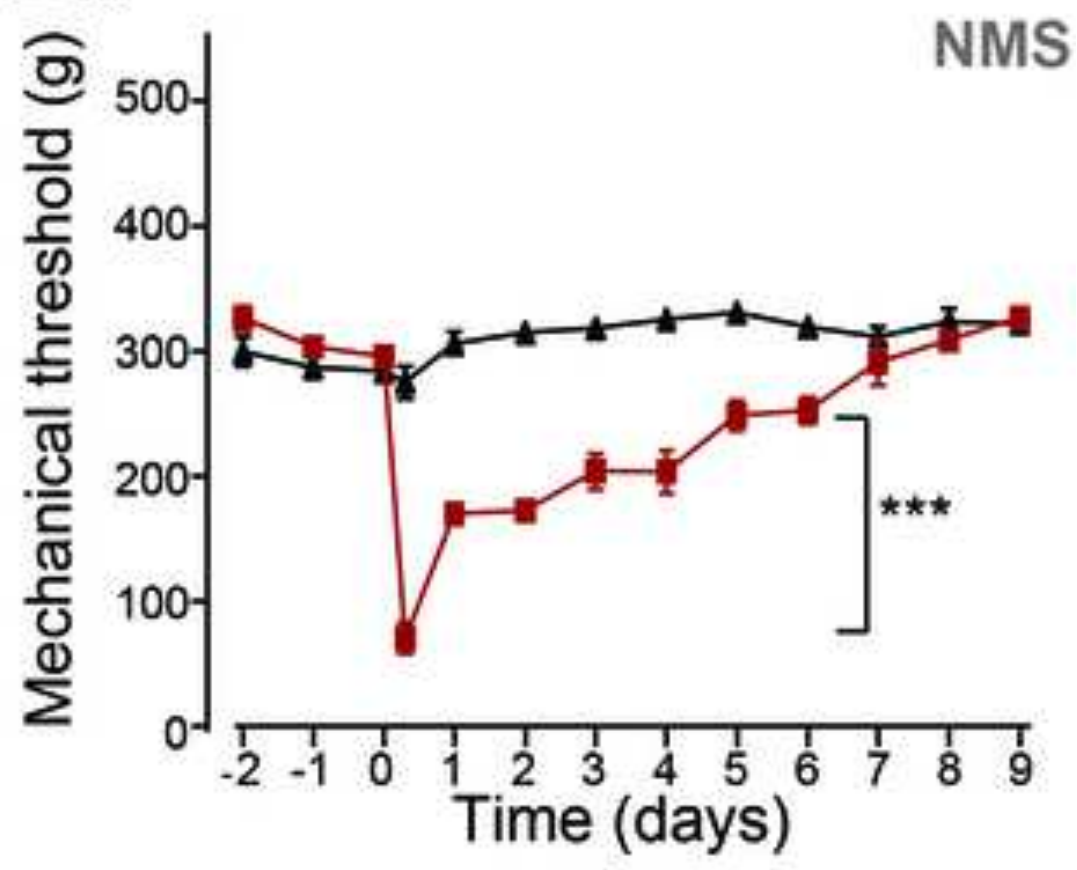

A2.

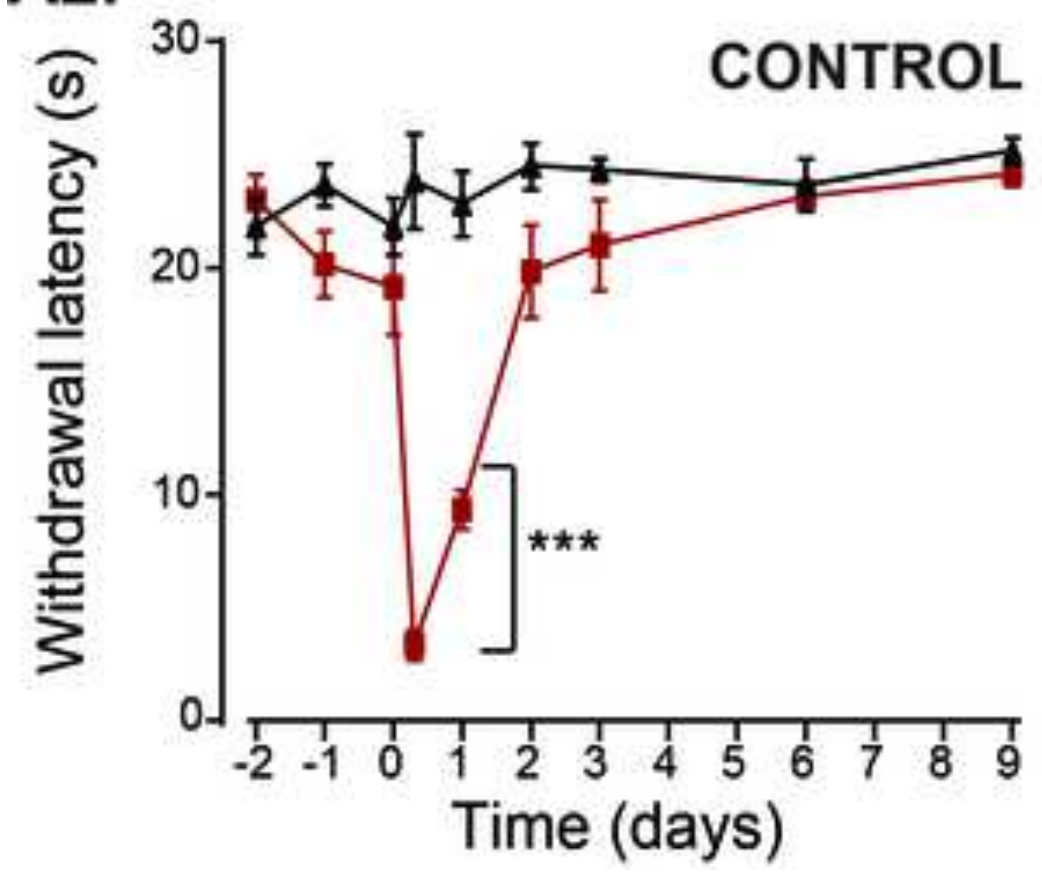

B2.

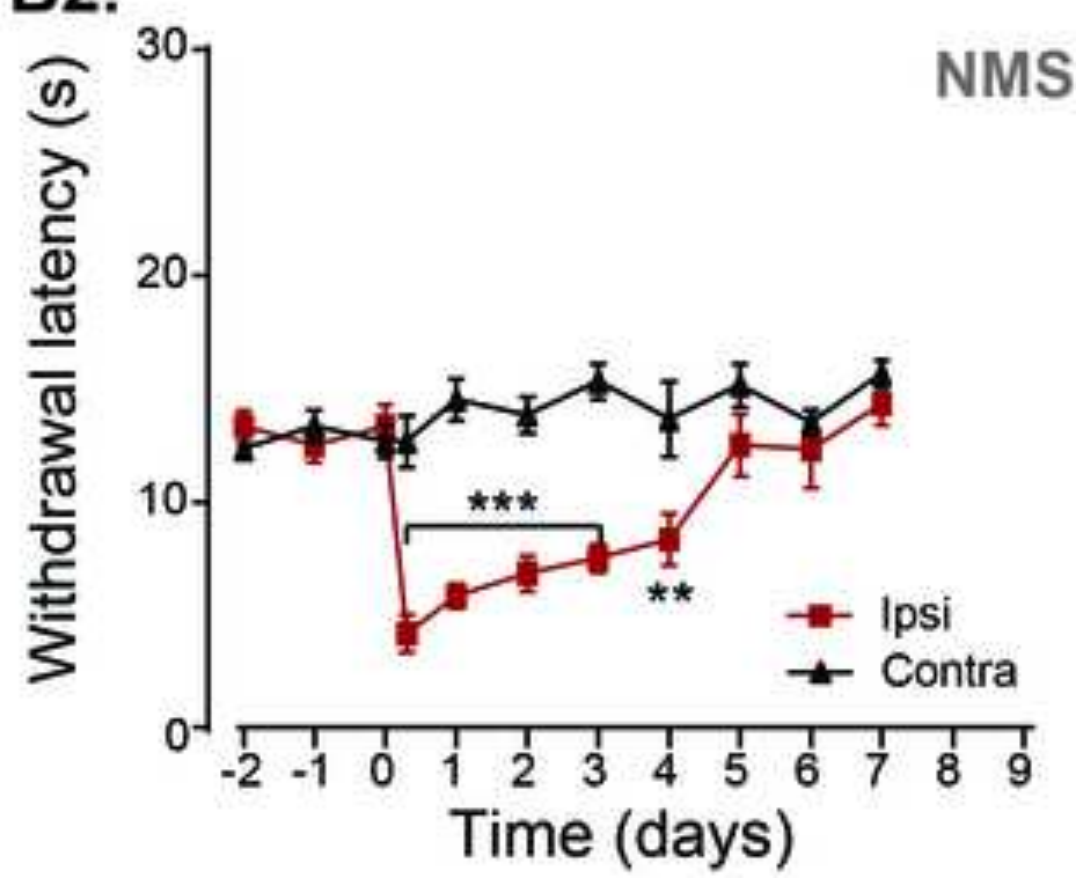




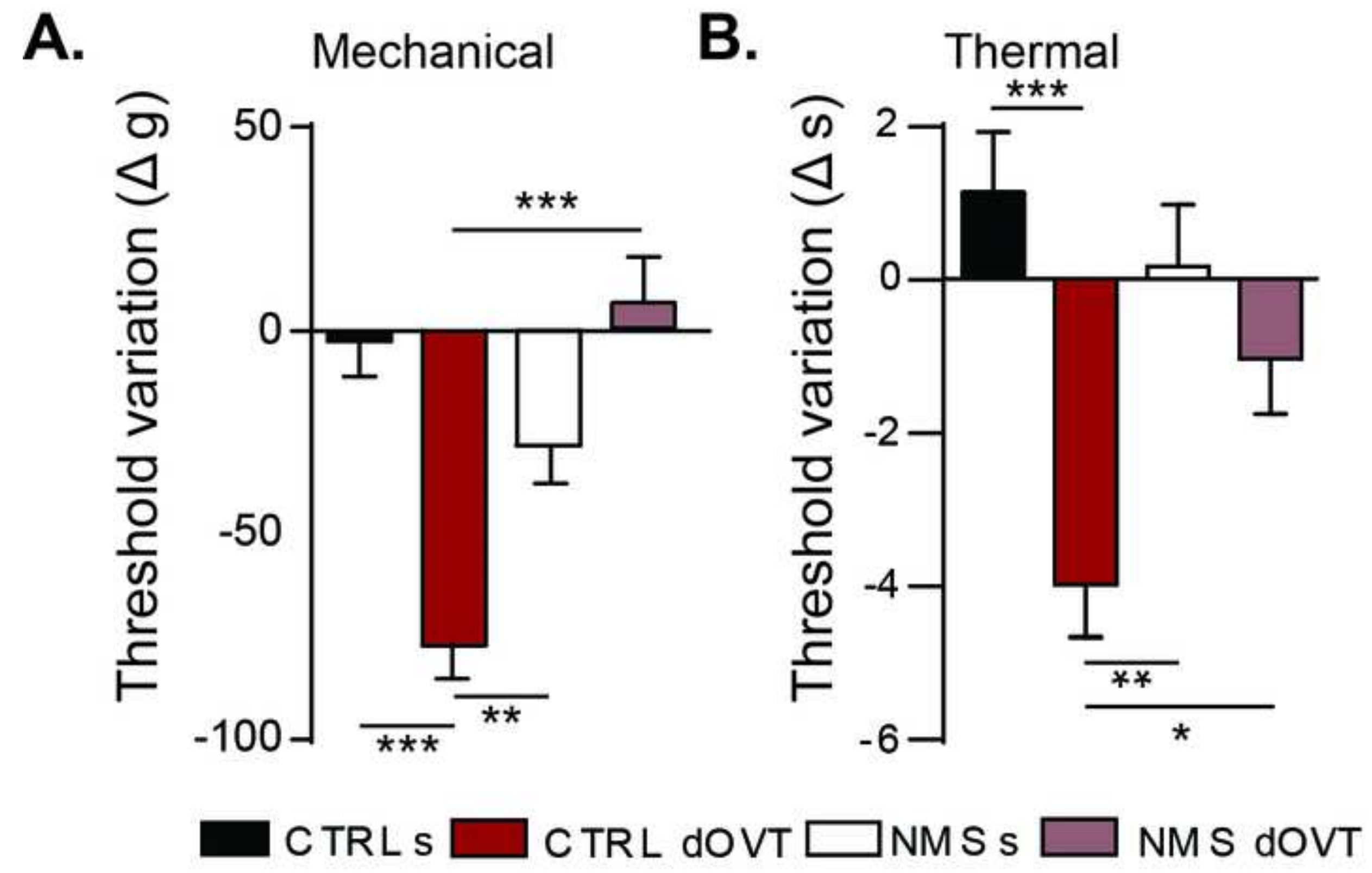



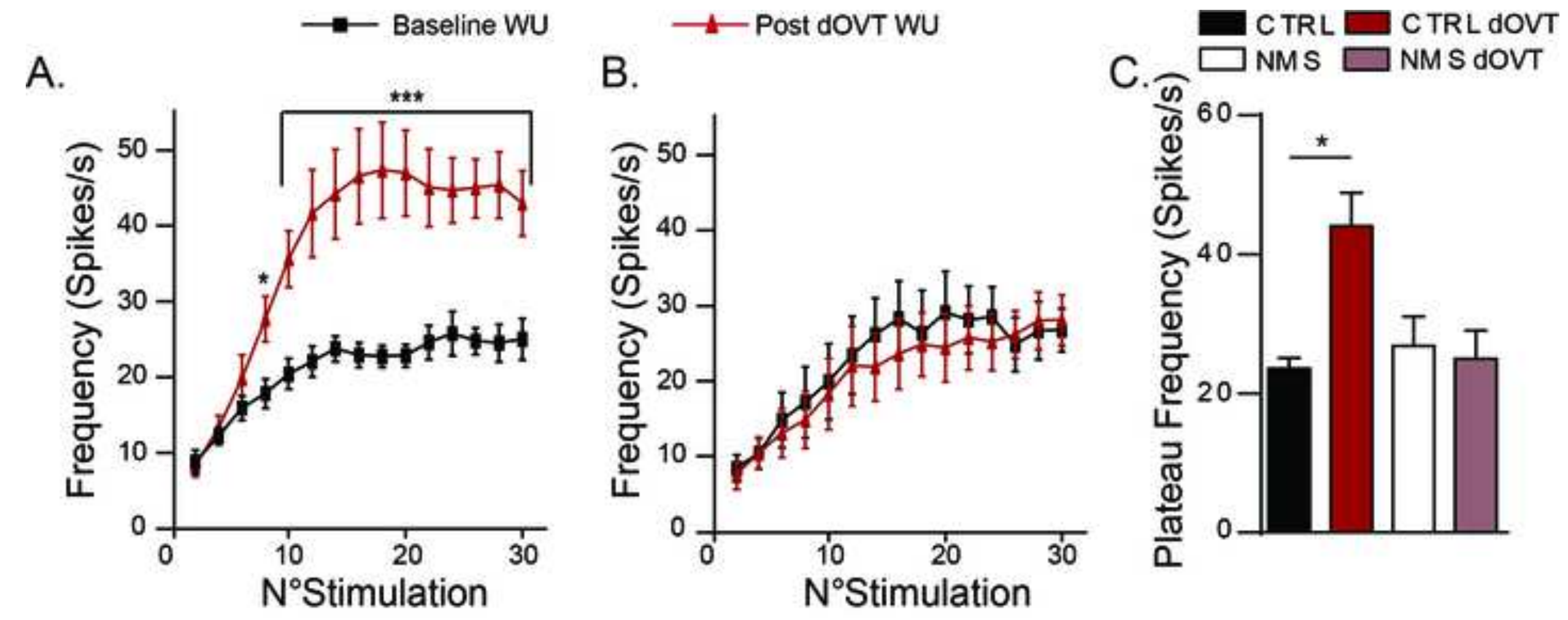
A.

B.

$\square$ CTRL $\square$ NMS

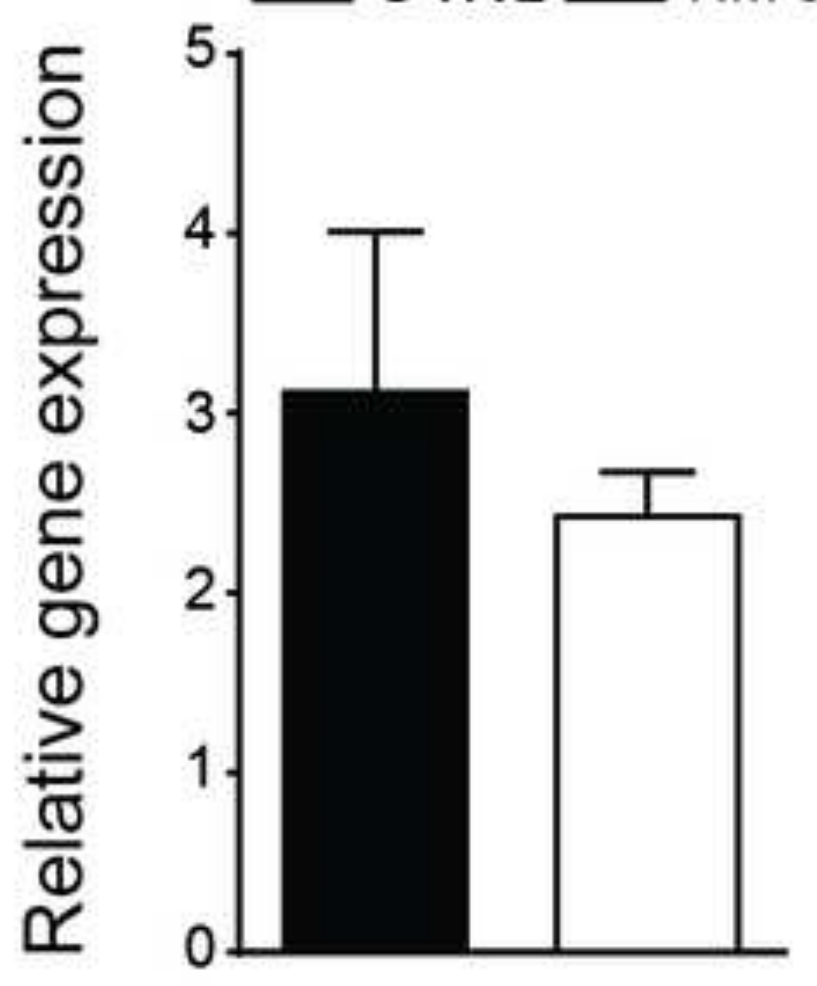

$-O-S A L-\square-$ TGOT $\triangle-$ OT

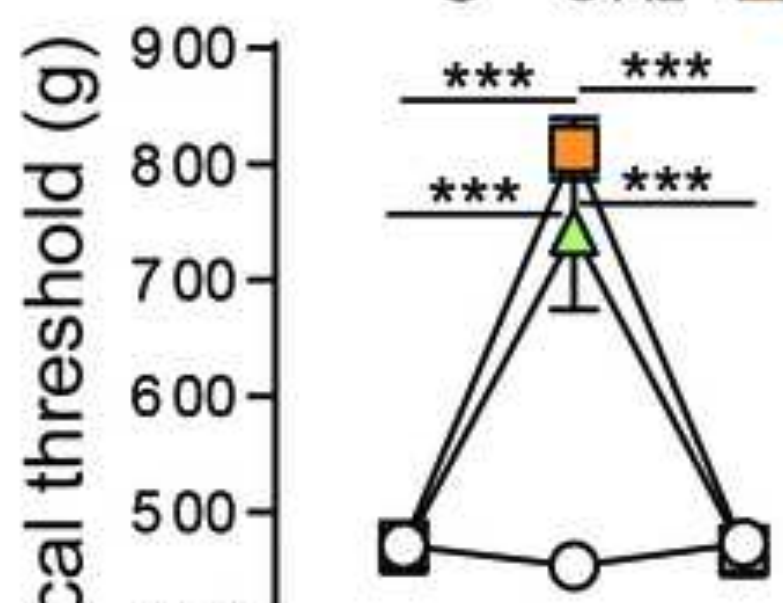

$900-$

을 $400-$

它 $300-$

$\sum_{\Sigma} 200$

CONTROL $200-$
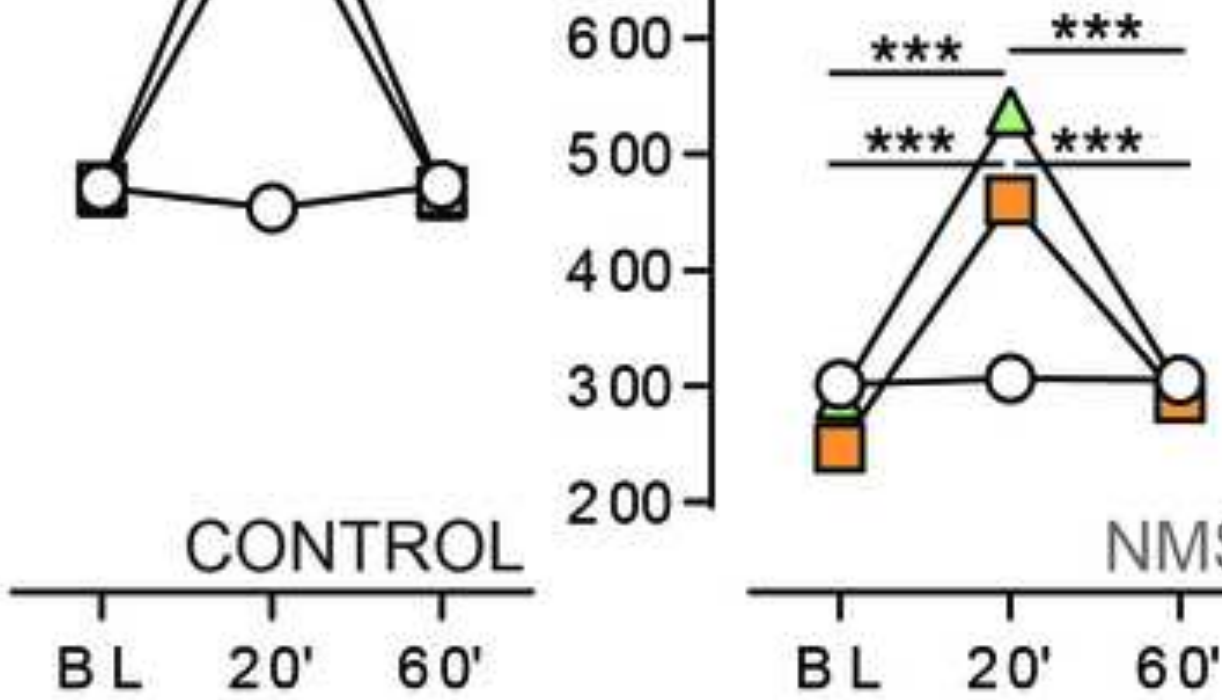

NMS

Time after i.t injection (minutes) 
A.

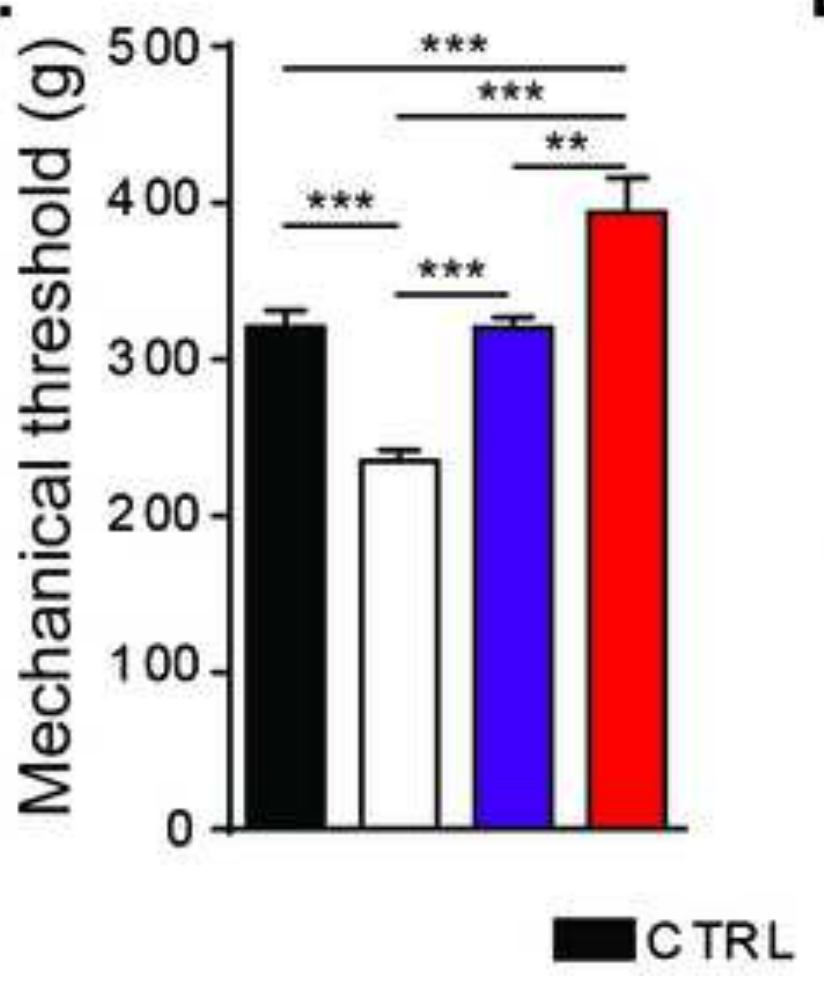

B.

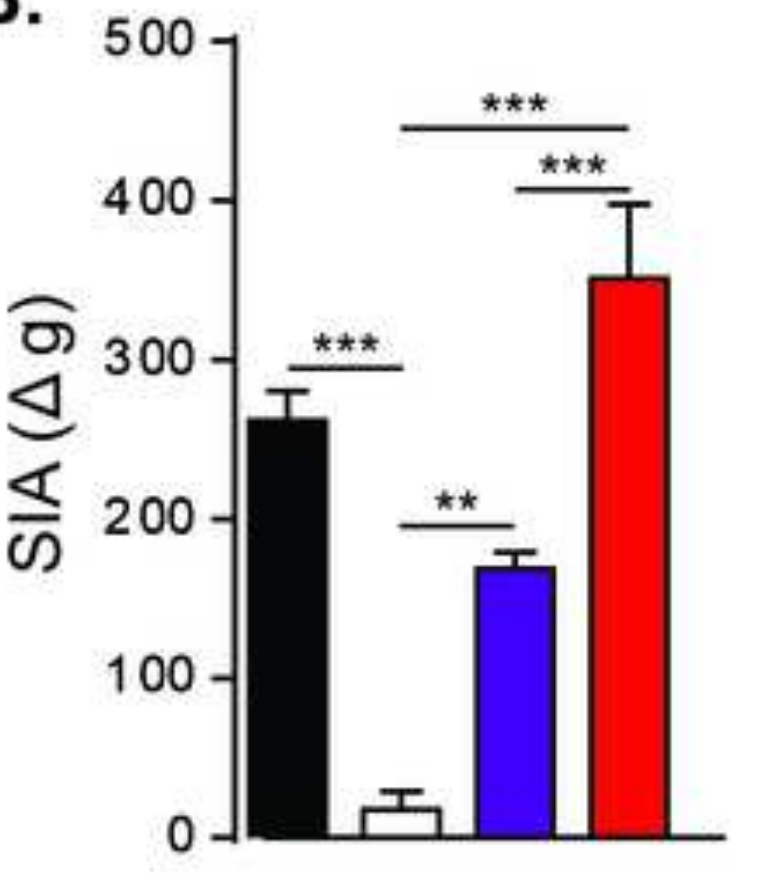

C.

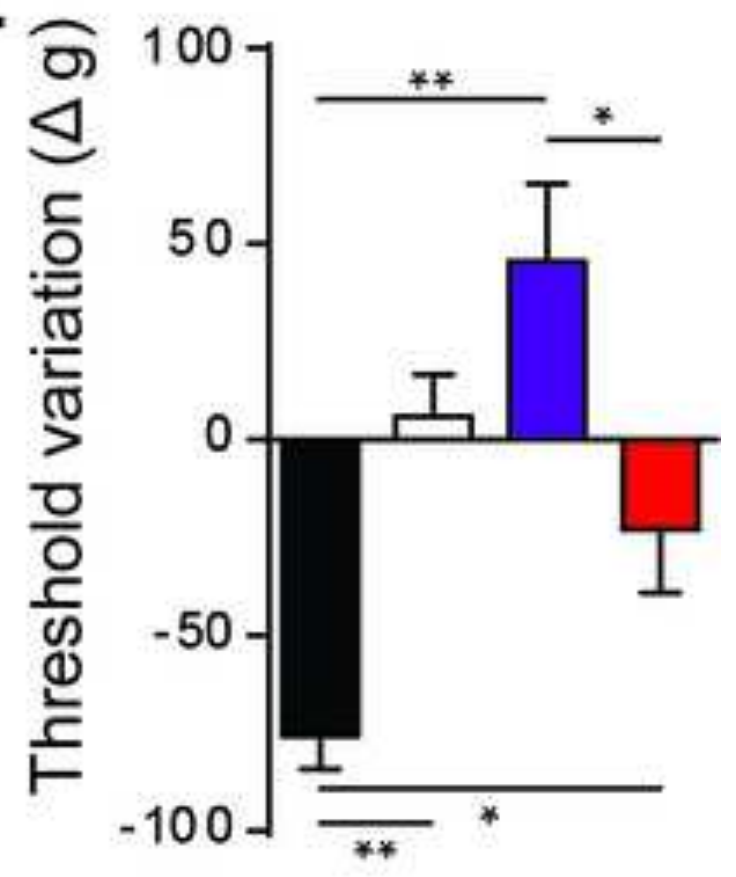

$\square$ NMS $\square$ NMS OT $\square$ NMS AP 
A.

\begin{tabular}{|l|c|c|c|c|c|}
\cline { 2 - 6 } \multicolumn{1}{c|}{} & P7 & P14 & P24 & P45 & P100 \\
\hline HDAC 1 & $=$ & $=$ & ++ & ++ & $=$ \\
\hline HDAC 2 & $=$ & $=$ & $=$ & $=$ & $=$ \\
\hline HDAC 3 & ND & $=$ & $=$ & ND & ND \\
\hline HDAC 4 & ND & $=$ & $=$ & ND & ND \\
\hline HDAC 5 & $=$ & $=$ & $=$ & $=$ & $=$ \\
\hline HDAC 6 & ND & $=$ & $=$ & ND & ND \\
\hline HDAC 7 & $=$ & $=$ & ++ & ++ & +++ \\
\hline HDAC 8 & $=$ & $=$ & $=$ & ++ & $=$ \\
\hline HDAC 9 & ND & $=$ & $=$ & ND & ND \\
\hline
\end{tabular}

C.

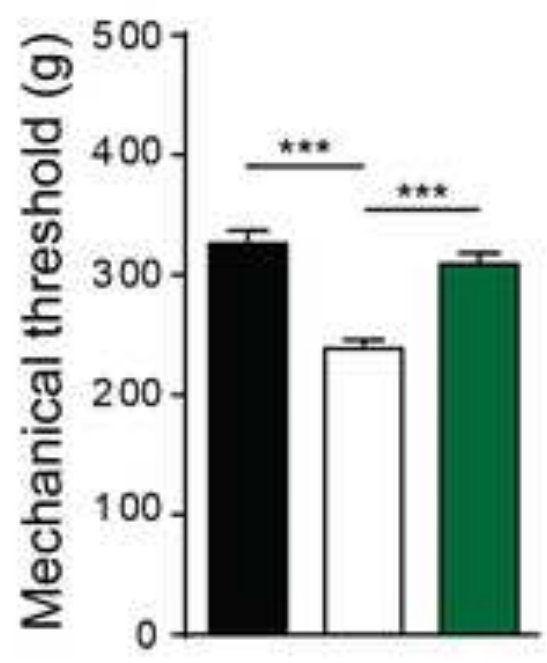

B.

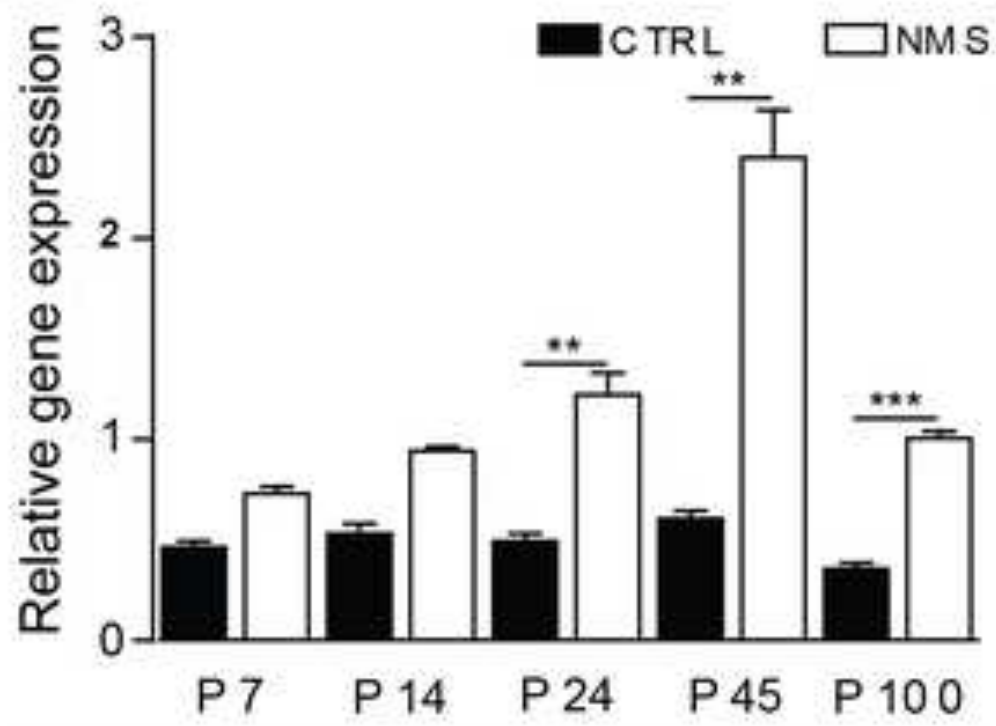

E.

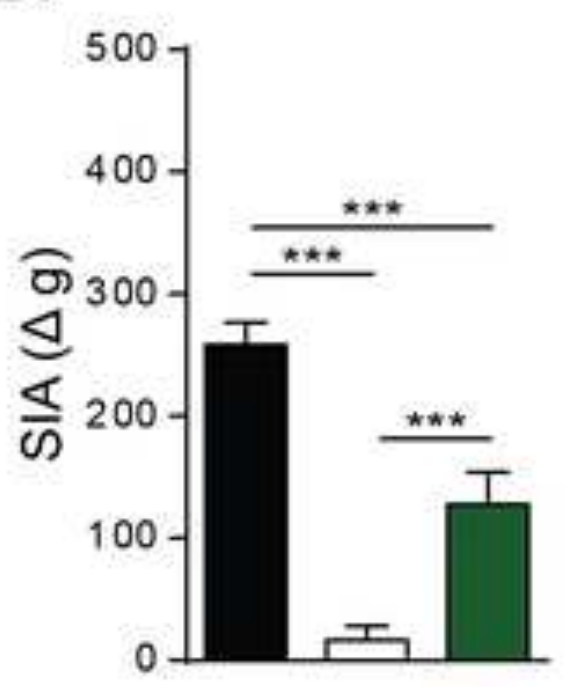

$\square$ CTRL $\square$ NMS $\square$ NMS SAHA 
A.

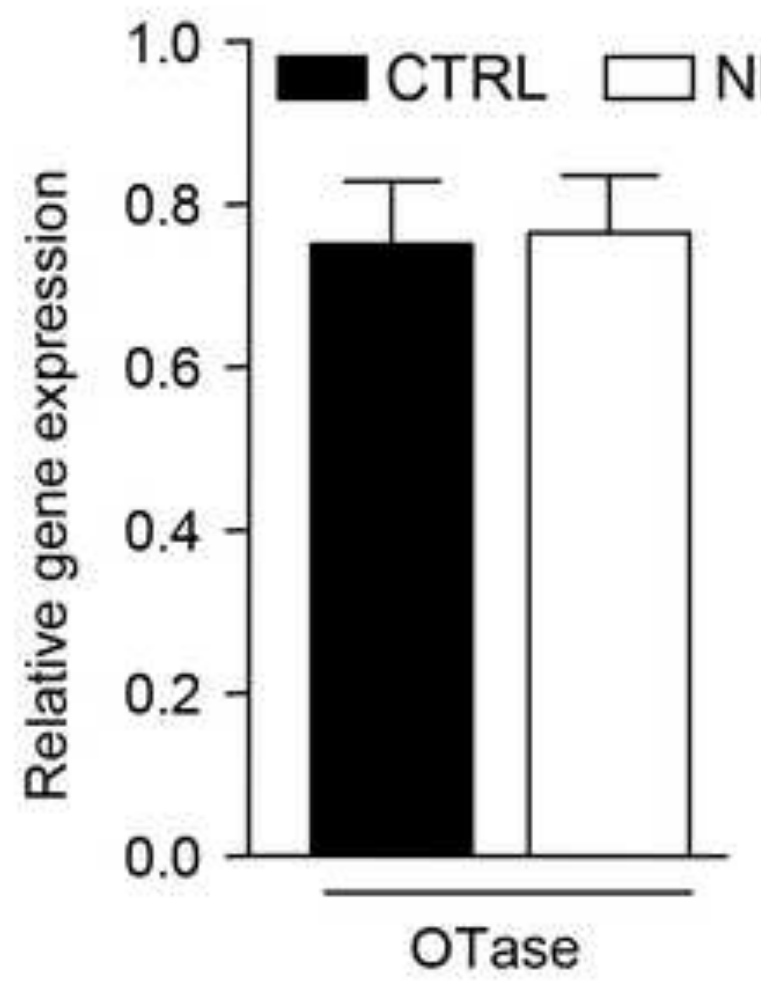

B.

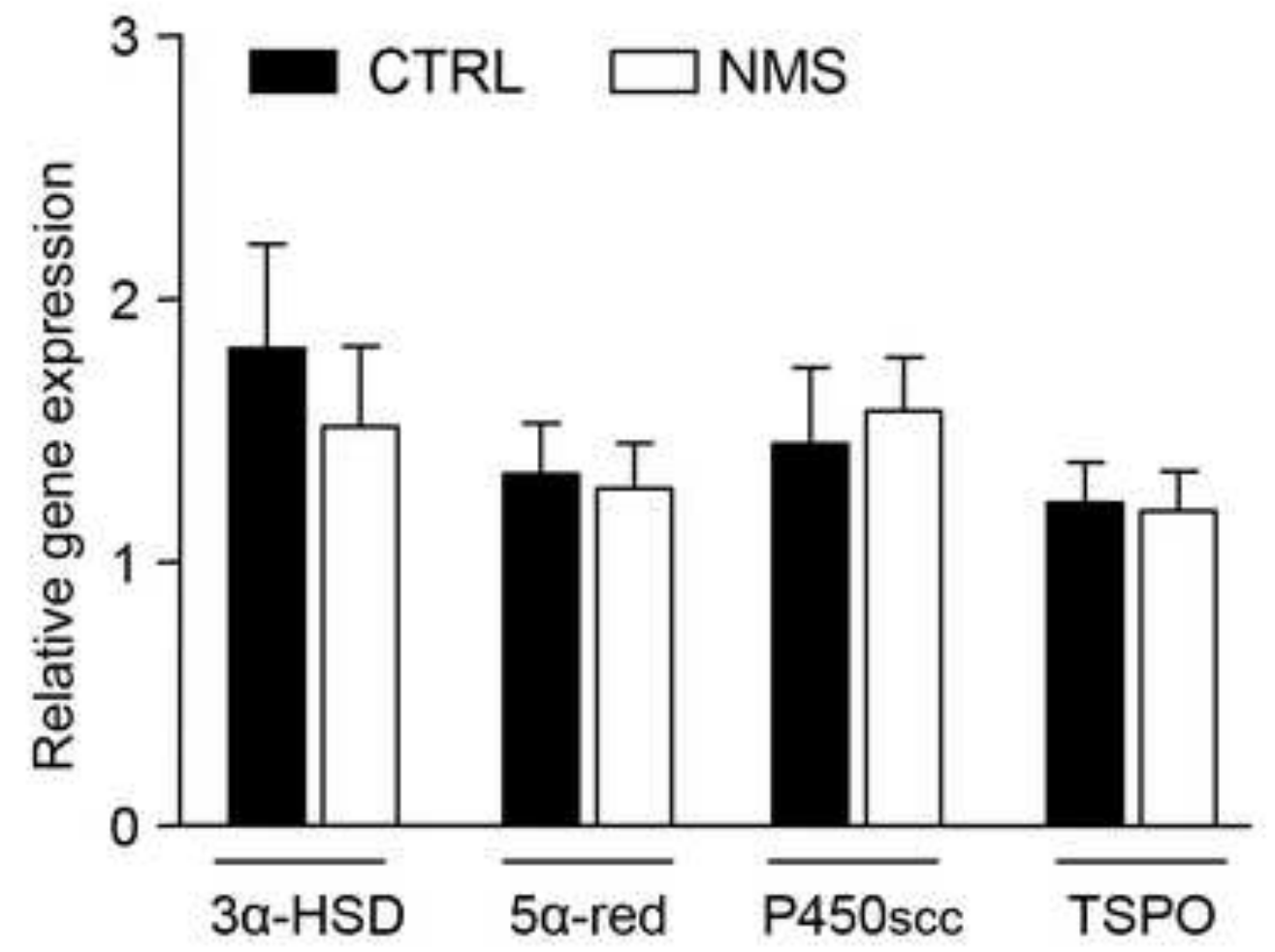




\begin{tabular}{|c|c|c|c|c|}
\hline Name & $\begin{array}{l}\text { Reference } \\
\text { sequence }\end{array}$ & $\begin{array}{c}\text { Amplicon } \\
\text { region }\end{array}$ & Forward sequence $\left(5^{\prime}\right.$ to $\left.3^{\prime}\right)$ & Reverse sequence (5' to $3^{\prime}$ ) \\
\hline $3 a-H S D$ & NM138547 & $330-423$ & ACCAGAGCTGGTCCGAACTT & CAAAGCCATTGGGAAATGAA \\
\hline 5a-red II & NM022711 & $49-146$ & TGCAGATTGTCTGCCATCAG & GTAACTGGCGGGTTTCCCTA \\
\hline HDAC 1 & NM001025409 & $247 \cdot 344$ & CGTTCTATTCGCCCAGATAAC & AACTCAAACAAGCCATCAAAT \\
\hline HDAC 2 & NM053447 & $1415-1510$ & AATTTCCATTCGAGCATCAGA & TCAGCAACATTCCTACGACCT \\
\hline HDAC 3 & NM053448 & $1238 \cdot 1346$ & GGGGTCCTGAGGAGAACTAC & CATCCATGCTGCTCTTAAATC \\
\hline HDAC 4 & NM053449 & $986-1089$ & ATGTACGACGCCAAAGATGA & CTGCTCCGTCTCTCAGCTACT \\
\hline HDAC 5 & NM053450 & $2084-2184$ & GAGGAGGACTGCATTCAGGTC & GCGAACAACTTTITGTAACCA \\
\hline HDAC 6 & KYO09929 & $1929 \cdot 2015$ & AATCGTGGATTGGGATGTTC & CGGTGCAGGGACACGTATAAT \\
\hline HDAC 7 & AF 321135 & $472-564$ & CСCCTGCGTAAAACAGTGTCT & CTTCCTGAGCAGGGGATT \\
\hline HDAC 8 & NM001126373 & $921-1023$ & GACTGTGTCCCTGCACAAGTT & GGGCACATTGACACTGTAGT \\
\hline HDAC 9 & NMO01200045 & $1666-1775$ & CCTGGAGAAGCAGAAGCAATA & ССТССTCTGCCTCTTCAAGAT \\
\hline HPRT & NM012583 & $558-647$ & GGTGAAAAGGACCTCTCGAA & TCAAGGGCATATCCAACAACA \\
\hline OT & NM012996 & $80-164$ & СTCTGACCTCCGCCTGCTA & GGAAGACACTTGCGCATATCC \\
\hline OTR & NM012871 & $732-825$ & TCGCCGTCTACATTGTACCG & GCTGCCGTCTTGAGTCTCAG \\
\hline OTase & NMO01113403 & $1934-2029$ & CCAGAGAAAGGGGACTGAGC & TGCCAAAGGTGGCTTGTATC \\
\hline P450sec & NM017286 & $679-780$ & GATGTTGGAGGAGATCGTGGA & AAGTCTGGAGGCATGTTGAGC \\
\hline TSPO & NM012515 & $152-243$ & ATGGGAGCCTACTTTGTGCGT & GGAGCGAGTGTCCAGCGA \\
\hline
\end{tabular}

